\title{
USDA Agricultural Marketing Service
}

\section{U.S. DEPARTMENT OF AGRICULTURE}

Quarter 4, October-December, 2020

Published August 2021 www.ams.usda.gov/services/transportation-analysis/agrta/ https://agtransport.usda.gov/

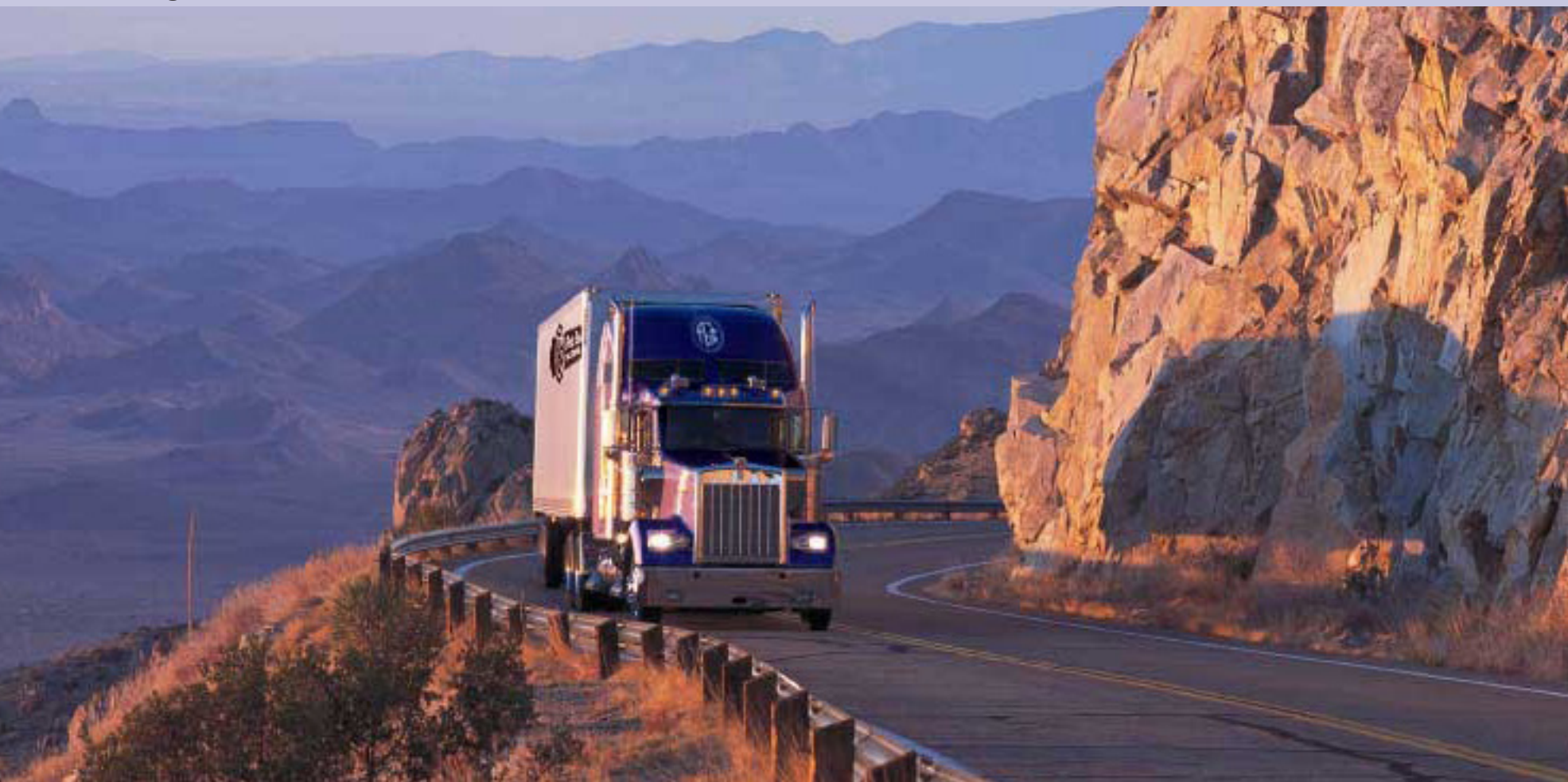

Agricultural Refrigerated Truck Quarterly

a quarterly publication of the Agricultural Marketing Service

\section{CONTENTS}

National Summary

Quarterly Overview 2

U.S. Truck Rates

2

Truck Rates for Selected Routes

U.S. Diesel Fuel Prices

3
Truck Rates.
Quarterly Truck Availability. Reported U.S. Shipments .8 Reported Shipments by Selected Commodities. 11

Regional Markets. .12

U.S.-Mexico Border 12 California 16
PNW 19

Arizona .. 22

Florida. 25

Additional Information .................28

Terms and References 28

Contact Us 29 


\section{NATIONAL SUMMARY Quarterly Overview}

Fruit and Vegetable Shipments

During fourth quarter 2020, reported U.S. truck shipments of fresh produce were 9.01 million tons, 1 percent lower than the previous quarter but 3 percent higher than the same quarter last year.

Also, in the fourth quarter, shipments from the Mexico border were 2.68 million tons, higher than those from any other reported origin and accounting for 30 percent of the total reported shipments of fresh fruit and vegetables. The remainder of the top five regions by volume are as follows: shipments from California, were 2.34 million tons, representing 26 percent of the total, shipments from the Pacific Northwest (PNW) were 1.84 million tons, representing 20 percent of the total, movements from Arizona were 386,000 tons, representing 4 percent of the reported total, and finally, Florida shipments were 305,000 tons, representing 3 percent of the reported total.

These top five commodities accounted for 38 percent of the reported truck movements during fourth quarter 2020:

Potatoes (13 percent)

- Apples (10 percent)

Onions, dry (6 percent)

Oranges (5 percent)

Tomatoes (4 percent)

\section{Truck Rates}

The table below provides a snapshot of quarterly truck rates for U.S. produce shipments over four mileage categories-0-500; 501-1,500; 1,501-2,500; and 2,501+ miles. Please note the U.S. average truck rates provided below were calculated using weighted regional rates and volumes.

Average U.S. Truck Rates for Selected Routes Between 501 and 1,500 Miles (\$/Mile)

\begin{tabular}{|c|c|c|c|c|}
\hline & 0-500 miles & $501-1,500$ miles & $1,501-2,500$ miles & $2,501+$ miles \\
\hline Q4 2019 & 3.21 & 2.52 & 2.29 & 1.37 \\
\hline Q1 2020 & 2.73 & 2.56 & 2.36 & 1.60 \\
\hline Q2 2020 & 2.57 & 2.51 & 2.27 & 1.55 \\
\hline Q3 2020 & 2.95 & 2.56 & 2.53 & 1.44 \\
\hline Q4 2020 & 3.26 & 2.92 & 2.85 & 1.66 \\
\hline $\begin{array}{l}\text { Q4 Change from } \\
\text { Previous Quarter }\end{array}$ & $11 \%$ & $14 \%$ & $13 \%$ & $15 \%$ \\
\hline $\begin{array}{r}\text { Q4 Change from Same } \\
\text { Quarter Last Year }\end{array}$ & $2 \%$ & $16 \%$ & $24 \%$ & $21 \%$ \\
\hline
\end{tabular}

Source: AMS Transportation Services Division analysis of AMS Specialty Crops Program Market News data.

Note: Table values may not conform exactly due to rounding.

Note: The rates for 8 long-haul fruit and vegetable truck corridors are included in the national rate, weighted by commodity and origin volume.

Diesel Fuel

During fourth quarter 2020 , the U.S. diesel fuel price averaged $\$ 2.466$ per gallon-up 2 percent from the previous quarter but 19 percent lower than the same quarter last year. 


\section{U.S. Truck Rates}

Figure 1: Average Truck Rates for Selected Routes (\$/Mile)

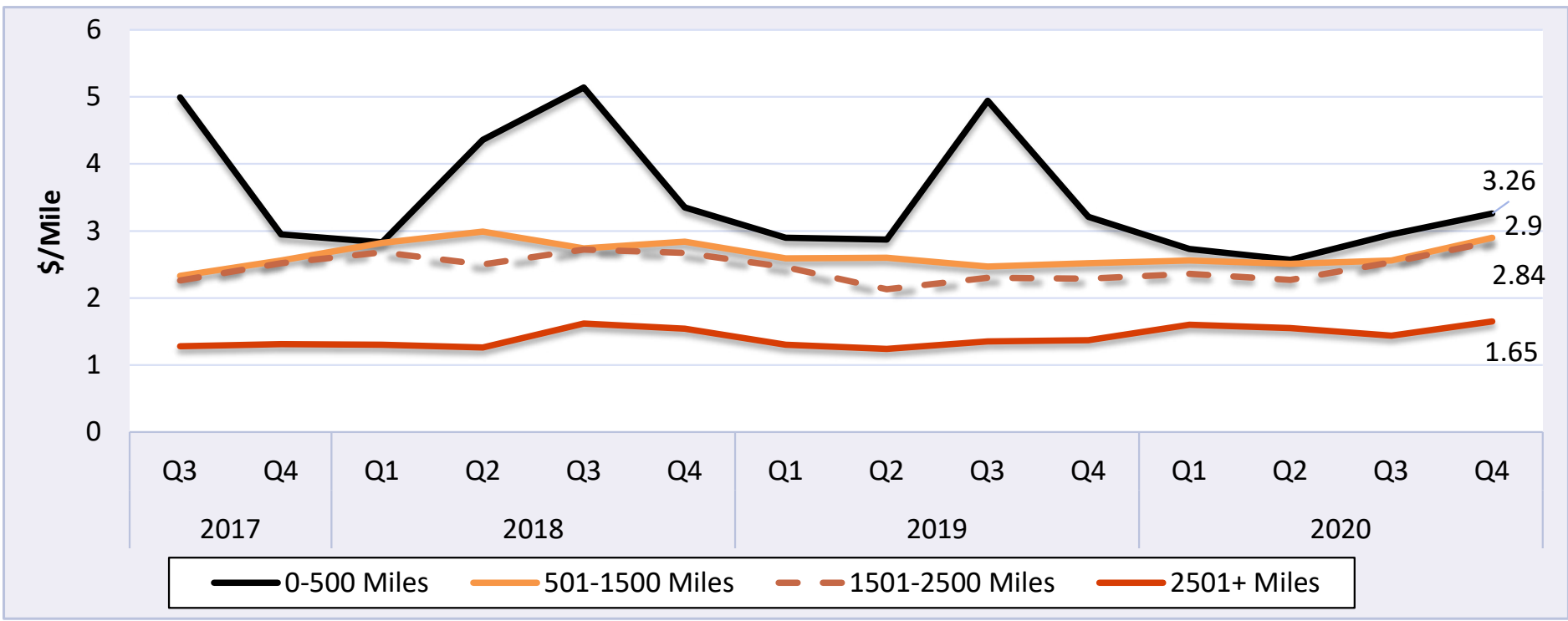

Source: AMS Transportation Services Division analysis of AMS Specialty Crops Program Market News data.

Note: The rates for 8 long-haul fruit and vegetable truck corridors are included in the national rates, weighted by commodity and origin volume.

Table 1: Average U.S. Truck Rates for Selected Routes Between 501 and 1,500 Miles (\$/Mile)

\begin{tabular}{|l|c|c|c|c|}
\hline Year & 1st Quarter & 2nd Quarter & 3rd Quarter & 4th Quarter \\
\hline $\mathbf{2 0 2 0}$ & 2.56 & 2.51 & 2.56 & 2.92 \\
\hline $\mathbf{2 0 1 9}$ & 2.59 & 2.60 & 2.47 & 2.52 \\
\hline $\mathbf{2 0 1 8}$ & 2.82 & 2.99 & 2.74 & 2.84 \\
\hline $\mathbf{2 0 1 7}$ & 1.86 & 2.41 & 2.33 & 2.56 \\
\hline $\mathbf{2 0 1 6}$ & 2.22 & 2.37 & 2.49 & 2.06 \\
\hline $\mathbf{2 0 1 5}$ & 2.47 & 2.63 & 2.59 & 2.36 \\
\hline $\mathbf{2 0 1 4}$ & 2.32 & 2.67 & 2.64 & 2.49 \\
\hline $\mathbf{2 0 1 3}$ & 2.24 & 2.60 & 2.62 & 2.27 \\
\hline $\mathbf{2 0 1 2}$ & 2.10 & 2.54 & 2.45 & 2.29 \\
\hline $\mathbf{2 0 1 1}$ & 2.02 & 2.60 & 2.77 & 2.26 \\
\hline $\mathbf{2 0 1 0}$ & 1.82 & 2.21 & 2.33 & 1.94 \\
\hline $\mathbf{2 0 0 9}$ & 1.85 & 1.99 & 2.02 & 1.86 \\
\hline $\mathbf{2 0 0 8}$ & 2.02 & 2.56 & 2.77 & 2.24 \\
\hline
\end{tabular}

Source: AMS Transportation Services Division analysis of AMS Specialty Crops Program Market News data. Note: The rates for 8 long-haul fruit and vegetable truck corridors are included in the national rate, weighted by commodity and origin volume.

Note: Table values may not conform exactly due to rounding. 
Table 2: Quarterly Rates for Key Origins by Month; 501-1,500 miles (\$/Mile)

\begin{tabular}{|r|c|c|c|c|c|c|}
\hline & \multicolumn{3}{|c|}{ 4th Quarter, 2020 } & \multicolumn{3}{c|}{ 3rd Quarter, 2020 } \\
\hline U.S. Origin & October & November & December & July & August & September \\
\hline Arizona & $\mathrm{n} / \mathrm{a}$ & $\mathrm{n} / \mathrm{a}$ & 4.09 & 3.17 & $\mathrm{n} / \mathrm{a}$ & $\mathrm{n} / \mathrm{a}$ \\
\hline California & 3.24 & 4.34 & 3.84 & 3.01 & 2.98 & 3.15 \\
\hline Florida & 2.33 & 2.27 & 2.53 & $\mathrm{n} / \mathrm{a}$ & $\mathrm{n} / \mathrm{a}$ & $\mathrm{n} / \mathrm{a}$ \\
\hline Great Lakes & 3.84 & 3.93 & 3.94 & 3.40 & 3.49 & 3.73 \\
\hline Mexico-Arizona & 2.47 & 2.98 & 2.94 & 2.23 & 1.70 & $\mathrm{n} / \mathrm{a}$ \\
\hline Mexico-Texas & 2.66 & 2.79 & 3.13 & 2.32 & 2.26 & 2.44 \\
\hline New York & 5.05 & 4.80 & 4.80 & $\mathrm{n} / \mathrm{a}$ & $\mathrm{n} / \mathrm{a}$ & 5.29 \\
\hline Other & 2.52 & 2.52 & 2.52 & 2.41 & 2.39 & 2.46 \\
\hline PNW & 2.25 & 2.40 & 2.43 & 1.93 & 1.91 & 2.20 \\
\hline Southeast & 6.85 & 5.21 & 4.24 & 4.09 & 5.49 & 6.02 \\
\hline Texas & 3.34 & 3.48 & 3.86 & 2.54 & 2.81 & 3.06 \\
\hline
\end{tabular}

Source: AMS Transportation Services Division analysis of AMS Specialty Crops Program Market News data. Note: "n/a" indicates rates not available.

\section{Truck Rates for Selected Routes}

Table 3: Origin-Destination Truck Rates for Selected Routes, 4th Quarter 2020 (\$/Mile)

\begin{tabular}{|r|c|c|c|c|c|c|c|c|c|}
\hline U.S. Origin & Atlanta & Baltimore & Boston & Chicago & Dallas & Los Angeles & Miami & New York & Philadelphia \\
\hline Arizona & 3.28 & 3.14 & 3.03 & 3.10 & 4.09 & $\mathrm{n} / \mathrm{a}$ & 3.29 & 3.14 & 3.13 \\
\hline California & 3.22 & 3.16 & 3.03 & 3.19 & 3.90 & $\mathrm{n} / \mathrm{a}$ & 3.15 & 3.14 & 3.22 \\
\hline Florida & 3.19 & 2.39 & 2.45 & 1.93 & $\mathrm{n} / \mathrm{a}$ & $\mathrm{n} / \mathrm{a}$ & $\mathrm{n} / \mathrm{a}$ & 2.61 & 2.35 \\
\hline Great Lakes & 3.73 & 4.21 & 3.74 & 4.60 & 3.24 & $\mathrm{n} / \mathrm{a}$ & 3.07 & 4.38 & 4.00 \\
\hline $\begin{array}{r}\text { Mexico- } \\
\text { Arizona }\end{array}$ & 2.98 & 3.12 & 3.07 & 2.56 & 3.26 & 2.36 & 3.07 & 3.15 & 3.17 \\
\hline $\begin{array}{r}\text { Mexico- } \\
\text { Texas }\end{array}$ & 3.20 & 3.09 & 2.95 & 2.56 & 3.23 & 2.19 & 2.94 & 3.00 & 3.00 \\
\hline New York & 5.21 & 6.49 & 11.74 & 5.41 & $\mathrm{n} / \mathrm{a}$ & $\mathrm{n} / \mathrm{a}$ & 4.02 & $\mathrm{n} / \mathrm{a}$ & 8.95 \\
\hline Other & 3.03 & 2.48 & 3.07 & 2.41 & 3.70 & 2.17 & 2.60 & 2.91 & 2.21 \\
\hline PNW & 2.75 & 2.90 & 2.85 & 2.83 & 2.73 & 2.37 & 2.70 & 2.96 & 2.89 \\
\hline Southeast & 9.01 & 10.58 & 6.15 & 4.39 & 3.92 & $\mathrm{n} / \mathrm{a}$ & 5.23 & 7.64 & 8.56 \\
\hline Texas & 4.33 & 3.96 & 3.60 & 3.65 & 5.38 & 2.50 & 3.26 & 3.78 & 3.80 \\
\hline
\end{tabular}

Source: AMS Transportation Services Division analysis of AMS Specialty Crops Program Market News data.

Note: "n/a" indicates rates not available. 
Table 4: Origin-Destination Truck Rates for Selected Routes, 4th Quarter 2020 (\$/Truck)

\begin{tabular}{|r|c|c|c|c|c|c|c|c|c|}
\hline & \multicolumn{7}{|c|}{ Destination } \\
\hline U.S. Origin & Atlanta & Baltimore & Boston & Chicago & Dallas & Los Angeles & Miami & New York & Philadelphia \\
\hline Arizona & 6,888 & 8,163 & 8,788 & 6,288 & 5,313 & $\mathrm{n} / \mathrm{a}$ & 8,550 & 8,488 & 8,288 \\
\hline California & 7,220 & 8,602 & 9,230 & 6,717 & 5,756 & $\mathrm{n} / \mathrm{a}$ & 8,867 & 8,924 & 8,902 \\
\hline Florida & 1,147 & 2,376 & 3,424 & 2,026 & $\mathrm{n} / \mathrm{a}$ & $\mathrm{n} / \mathrm{a}$ & $\mathrm{n} / \mathrm{a}$ & 3,065 & 2,574 \\
\hline Great Lakes & 3,806 & 3,897 & 5,148 & 1,602 & 3,579 & $\mathrm{n} / \mathrm{a}$ & 5,546 & 4,435 & 3,919 \\
\hline $\begin{array}{r}\text { Mexico- } \\
\text { Arizona }\end{array}$ & 5,365 & 7,331 & 8,296 & 4,600 & 3,196 & 1,319 & 6,992 & 7,877 & 7,612 \\
\hline $\begin{array}{r}\text { Mexico- } \\
\text { Texas }\end{array}$ & 3,681 & 5,538 & 6,496 & 3,654 & 1,615 & 3,504 & 4,504 & 6,004 & 5,700 \\
\hline New York & 5,211 & 2,141 & 1,995 & 4,544 & $\mathrm{n} / \mathrm{a}$ & $\mathrm{n} / \mathrm{a}$ & 5,823 & $\mathrm{n} / \mathrm{a}$ & 2,059 \\
\hline Other & 2,478 & 4,446 & 6,603 & 2,279 & 1,961 & 2,018 & 5,339 & 4,298 & 4,068 \\
\hline PNW & 6,363 & 7,150 & 7,837 & 5,032 & 4,986 & 2,367 & 8,010 & 7,512 & 7,265 \\
\hline Southeast & 3,604 & 3,491 & 4,549 & 3,729 & 4,710 & $\mathrm{n} / \mathrm{a}$ & 4,030 & 3,974 & 3,680 \\
\hline Texas & 3,681 & 5,538 & 6,488 & 3,654 & 1,615 & 3,504 & 4,504 & 6,015 & 5,700 \\
\hline
\end{tabular}

Source: AMS Transportation Services Division analysis of AMS Specialty Crops Program Market News data.

\section{U.S. Diesel Fuel Prices}

The diesel fuel price provides a proxy for trends in U.S. truck rates. Diesel fuel is a significant component underlying truck rates.

\section{Figure 2: U.S. Average On-Highway Diesel Fuel Prices}

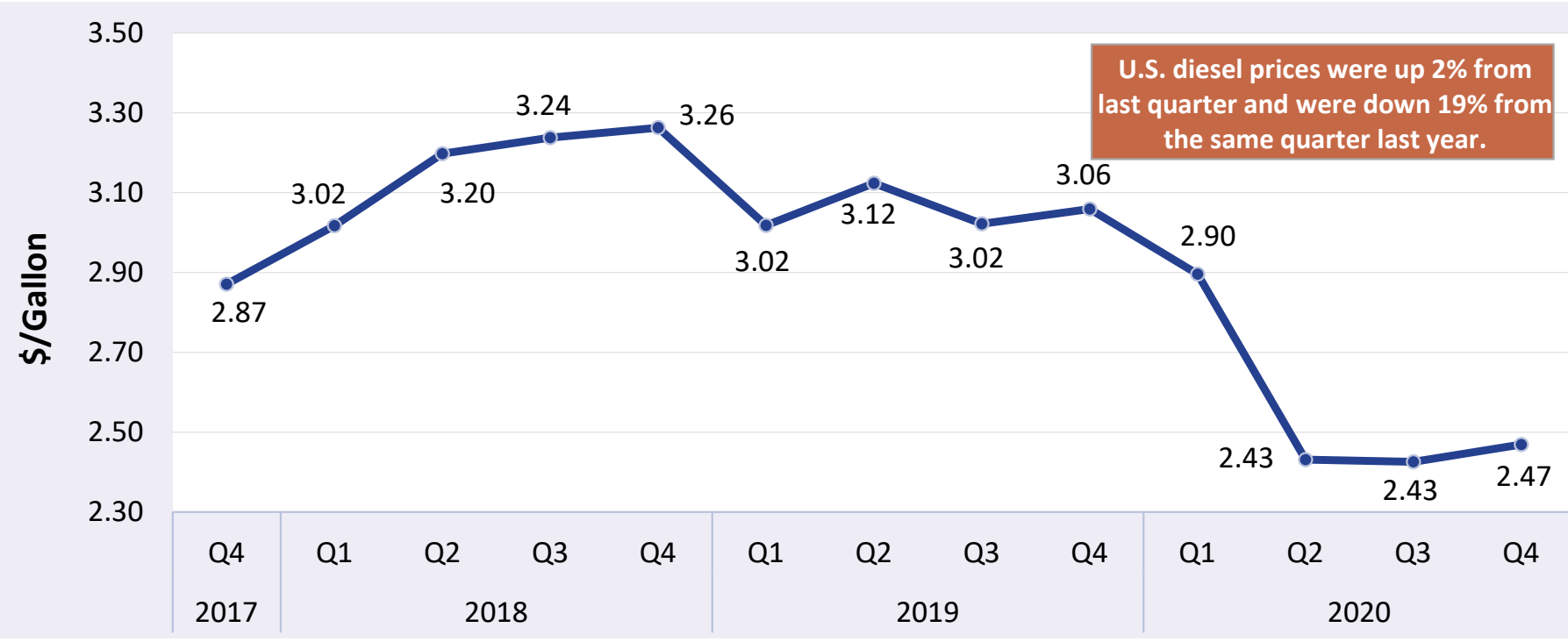

Source: AMS Transportation Services Division analysis of Energy Information Administration/U.S. Department of Energy data. 
Table 5: Average Diesel Fuel Prices (All Types)

\begin{tabular}{|r|c|c|c|}
\multicolumn{1}{|c|}{ Region } & $\begin{array}{c}\text { 4th Quarter 2020 Price } \\
\mathbf{\$} / \text { Gallon }\end{array}$ & $\begin{array}{c}\text { Change From Last } \\
\text { Quarter }\end{array}$ & Change From Last Year \\
\hline East Coast & 2.52 & $<1 \%$ & $-17 \%$ \\
\hline California & 3.28 & $1 \%$ & $-17 \%$ \\
\hline New England & 2.58 & $-2 \%$ & $-16 \%$ \\
\hline Central Atlantic & 2.72 & $1 \%$ & $-16 \%$ \\
\hline Lower Atlantic & 2.38 & $<1 \%$ & $-18 \%$ \\
\hline Gulf Coast & 2.22 & $2 \%$ & $-20 \%$ \\
\hline Midwest & 2.37 & $3 \%$ & $-20 \%$ \\
\hline Rocky Mountain & 2.45 & $4 \%$ & $-22 \%$ \\
\hline West Coast Except California & 2.65 & $3 \%$ & $-20 \%$ \\
\hline U.S. & 2.47 & $2 \%$ & $-19 \%$ \\
\hline
\end{tabular}

Source: AMS Transportation Services Division analysis of Energy Information Administration/U.S. Department of Energy data. Note: Table values may not conform exactly due to rounding.

\section{Relationship Between Diesel Fuel and Truck Rates}

The diesel fuel price provides a proxy for trends in U.S. truck rates. Diesel fuel is a significant expense for fruit and vegetable movements.

Figure 3: U.S. Average On-Highway Diesel Fuel Prices and Truck Rates

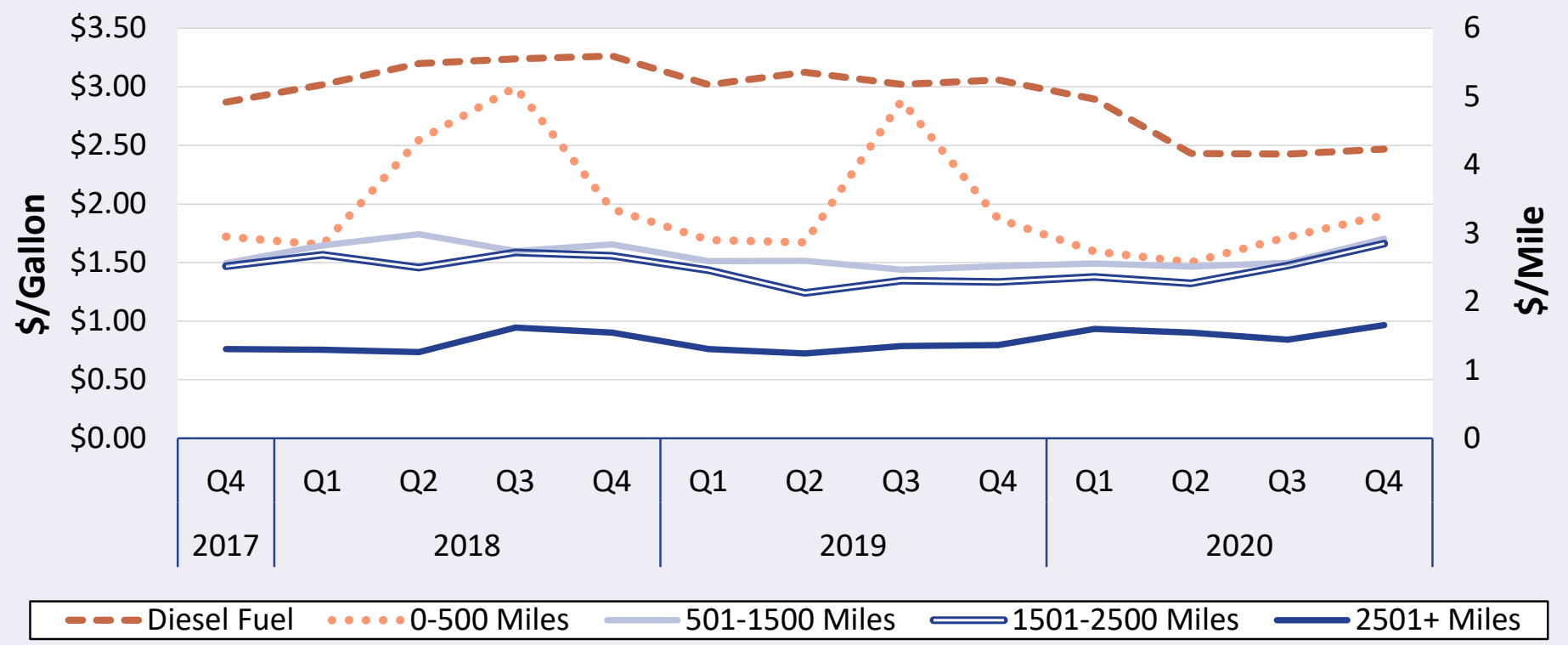

Sources: AMS Transportation Services Division analysis of AMS Specialty Crops Program Market News data and Energy Information Administration/ U.S. Department of Energy data.

Note: The rates for 8 long-haul fruit and vegetable truck corridors are included in the national rates, weighted by origin volume. 
Table 6: Average Diesel Fuel Prices and Truck Rates

\begin{tabular}{|c|c|c|c|c|c|c|c|}
\hline \multirow{3}{*}{ Year } & \multirow{3}{*}{ Quarter } & \multirow{3}{*}{$\begin{array}{l}\text { Diesel Fuel } \\
\text { (\$/gallon) }\end{array}$} & \multirow{3}{*}{$\begin{array}{c}\text { Truck Rates (\$/mile) } \\
\text { 501-1500 miles }\end{array}$} & \multicolumn{4}{|c|}{$\%$ Change From } \\
\hline & & & & \multicolumn{2}{|c|}{ Last Quarter } & \multicolumn{2}{|c|}{ Last Year } \\
\hline & & & & Diesel & Truck & Diesel & Truck \\
\hline 2017 & Q4 & 2.87 & 2.56 & $9 \%$ & $10 \%$ & $16 \%$ & $25 \%$ \\
\hline \multirow[t]{4}{*}{2018} & Q1 & 3.02 & 2.82 & $5 \%$ & $10 \%$ & $18 \%$ & $51 \%$ \\
\hline & Q2 & 3.20 & 2.99 & $6 \%$ & $6 \%$ & $25 \%$ & $24 \%$ \\
\hline & Q3 & 3.24 & 2.74 & $1 \%$ & $-8 \%$ & $23 \%$ & $18 \%$ \\
\hline & Q4 & 3.26 & 2.84 & $1 \%$ & $4 \%$ & $14 \%$ & $11 \%$ \\
\hline \multirow[t]{4}{*}{2019} & Q1 & 3.02 & 2.59 & $-8 \%$ & $-9 \%$ & $<1 \%$ & $-8 \%$ \\
\hline & Q2 & 3.12 & 2.60 & $4 \%$ & $<1 \%$ & $-2 \%$ & $-13 \%$ \\
\hline & Q3 & 3.02 & 2.47 & $-3 \%$ & $-5 \%$ & $-7 \%$ & $-10 \%$ \\
\hline & Q4 & 3.06 & 2.52 & $1 \%$ & $2 \%$ & $-6 \%$ & $-11 \%$ \\
\hline \multirow[t]{4}{*}{2020} & Q1 & 2.90 & 2.56 & $-5 \%$ & $2 \%$ & $-4 \%$ & $-1 \%$ \\
\hline & Q2 & 2.43 & 2.51 & $-16 \%$ & $-2 \%$ & $-22 \%$ & $-3 \%$ \\
\hline & Q3 & 2.43 & 2.56 & $>-1 \%$ & $2 \%$ & $-20 \%$ & $4 \%$ \\
\hline & Q4 & 2.47 & 2.92 & $2 \%$ & $14 \%$ & $-19 \%$ & $16 \%$ \\
\hline
\end{tabular}

Sources: AMS Transportation Services Division analysis of AMS Specialty Crops Program Market News data and Energy Information Administration/U.S. Department of Energy data.

Note: Table values may not conform exactly due to rounding. 


\section{Quarterly Truck Availability}

Table 7: U.S. Fresh Fruit and Vegetable Truck Availability

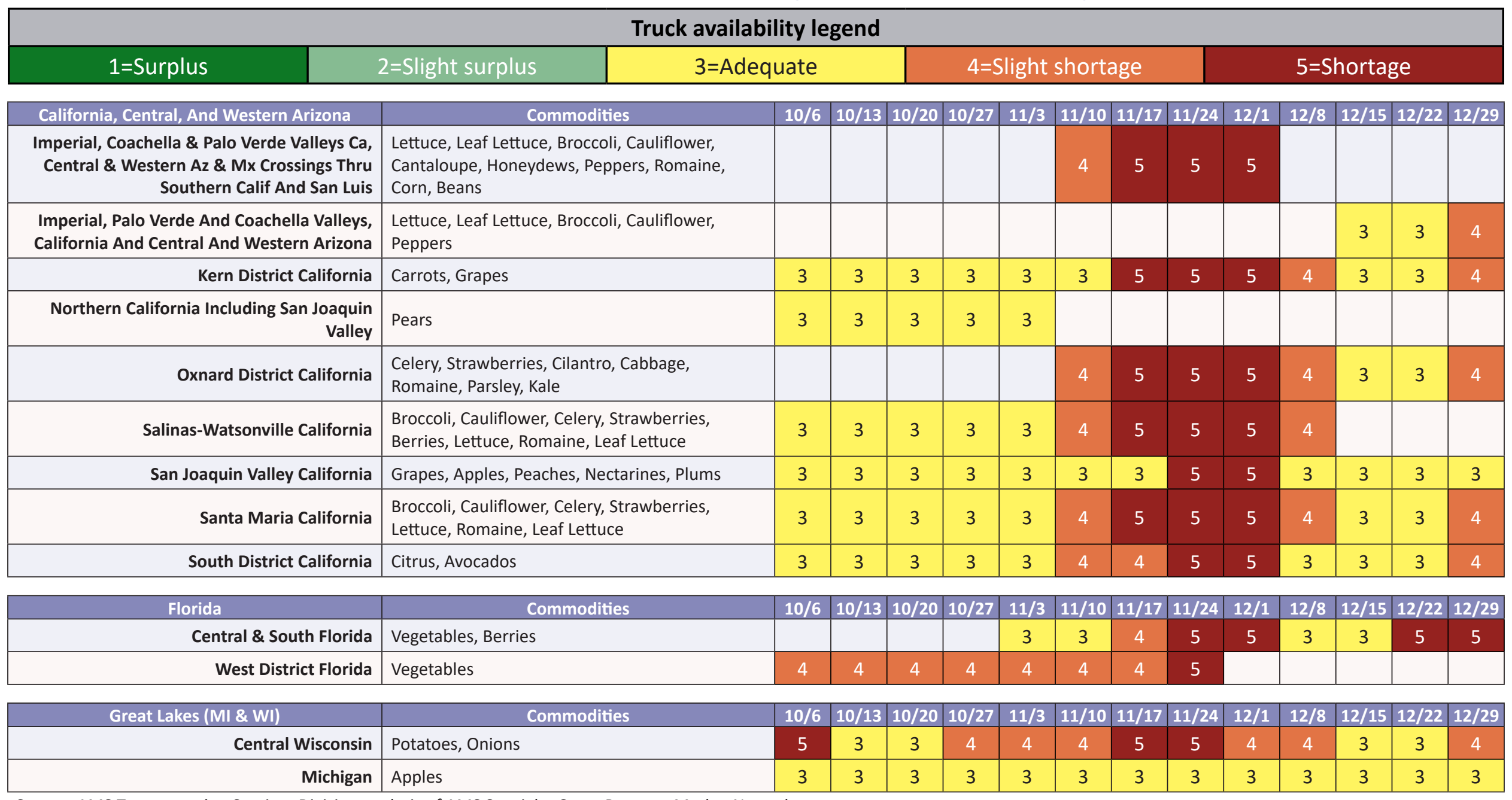

Source: AMS Transportation Services Division analysis of AMS Specialty Crops Program Market News data.

Note: Empty cells were not reported. 
Table 7, continued: U.S. Fresh Fruit and Vegetable Truck Availability

\begin{tabular}{|c|c|c|c|c|}
\hline \multicolumn{4}{|c|}{ Truck availability legend } \\
\hline 1=Surplus & 2=Slight surplus & 3=Adequate & 4=Slight shortage & 5=Shortage \\
\hline
\end{tabular}

\begin{tabular}{|c|c|c|c|c|c|c|c|c|c|c|c|c|c|c|}
\hline Pacific Northwest (ID, OR, \&, WA) & Commodities & $10 / 6$ & $10 / 13$ & $10 / 20$ & $10 / 27$ & $11 / 3$ & $11 / 10$ & $11 / 17$ & $11 / 24$ & $12 / 1$ & $12 / 8$ & $12 / 15$ & $12 / 22$ & $12 / 29$ \\
\hline Columbia Basin Washington & Potatoes, Onions & 5 & 5 & 5 & 5 & 5 & 5 & 5 & 5 & 5 & 5 & 4 & 4 & 4 \\
\hline Idaho And Malheur County, Oregon & Onions & 5 & 5 & 5 & 5 & 5 & 5 & 5 & 5 & 5 & 5 & 4 & 4 & 4 \\
\hline Upper Valley, Twin Falls-Burley District Idaho & Potatoes & 5 & 5 & 5 & 5 & 5 & 5 & 5 & 5 & 4 & 4 & 4 & 4 & 5 \\
\hline $\begin{array}{r}\text { Yakima Valley \& Wenatchee District } \\
\text { Washington }\end{array}$ & Apples, Pears & 3 & 3 & 3 & 4 & 5 & 5 & 5 & 5 & 4 & 3 & 3 & 3 & 4 \\
\hline
\end{tabular}

\begin{tabular}{|c|c|c|c|c|c|c|c|c|c|c|c|c|c|c|}
\hline Southeast (GA, SC, \& NC) & Commodities & $10 / 6$ & $10 / 13$ & $10 / 20$ & $10 / 27$ & $11 / 3$ & $11 / 10$ & $11 / 17$ & $11 / 24$ & $12 / 1$ & $12 / 8$ & $12 / 15$ & $12 / 22$ & $12 / 29$ \\
\hline Eastern North Carolina & Sweet Potatoes & 4 & 4 & 4 & 4 & 4 & 4 & 5 & 5 & 3 & 4 & 4 & 5 & 5 \\
\hline Texas and Oklahoma & Commodities & $10 / 6$ & $10 / 13$ & $10 / 20$ & $10 / 27$ & $11 / 3$ & $11 / 10$ & $11 / 17$ & $11 / 24$ & $12 / 1$ & $12 / 8$ & $12 / 15$ & $12 / 22$ & $12 / 29$ \\
\hline Texas & Watermelons & 3 & 3 & 3 & 4 & 4 & 4 & 4 & 4 & 5 & 3 & 3 & 5 & 5 \\
\hline
\end{tabular}

\begin{tabular}{|c|c|c|c|c|c|c|c|c|c|c|c|c|c|c|}
\hline U.S.-Mexico border & Commodities & $10 / 6$ & $10 / 13$ & $10 / 20$ & $10 / 27$ & $11 / 3$ & $11 / 10$ & $11 / 17$ & $11 / 24$ & $12 / 1$ & $12 / 8$ & $12 / 15$ & $12 / 22$ & $12 / 29$ \\
\hline Nogales, Arizona & $\begin{array}{l}\text { Cucumbers, Eggplant, Squash, Cantaloupe, } \\
\text { Honeydew, Watermelon }\end{array}$ & 3 & 3 & 4 & 4 & 4 & 4 & 5 & 5 & 3 & 3 & 4 & 5 & 5 \\
\hline Texas & $\begin{array}{l}\text { Limes, Mangos, Tomatoes, Broccoli, Mixed Fruit } \\
\text { and Vegetables }\end{array}$ & 3 & 3 & 3 & 4 & 4 & 4 & 4 & 4 & 5 & 3 & 3 & 5 & 5 \\
\hline
\end{tabular}

Source: AMS Transportation Services Division analysis of AMS Specialty Crops Program Market News data.

Note: Empty cells were not reported. 


\section{Agricultural Refrigerated Truck Quarterly \\ Quarter 4, 2020}

\section{Reported U.S. Shipments}

Figure 4: Reported U.S. Fruit and Vegetable Shipments (1,000 Tons)

12,000

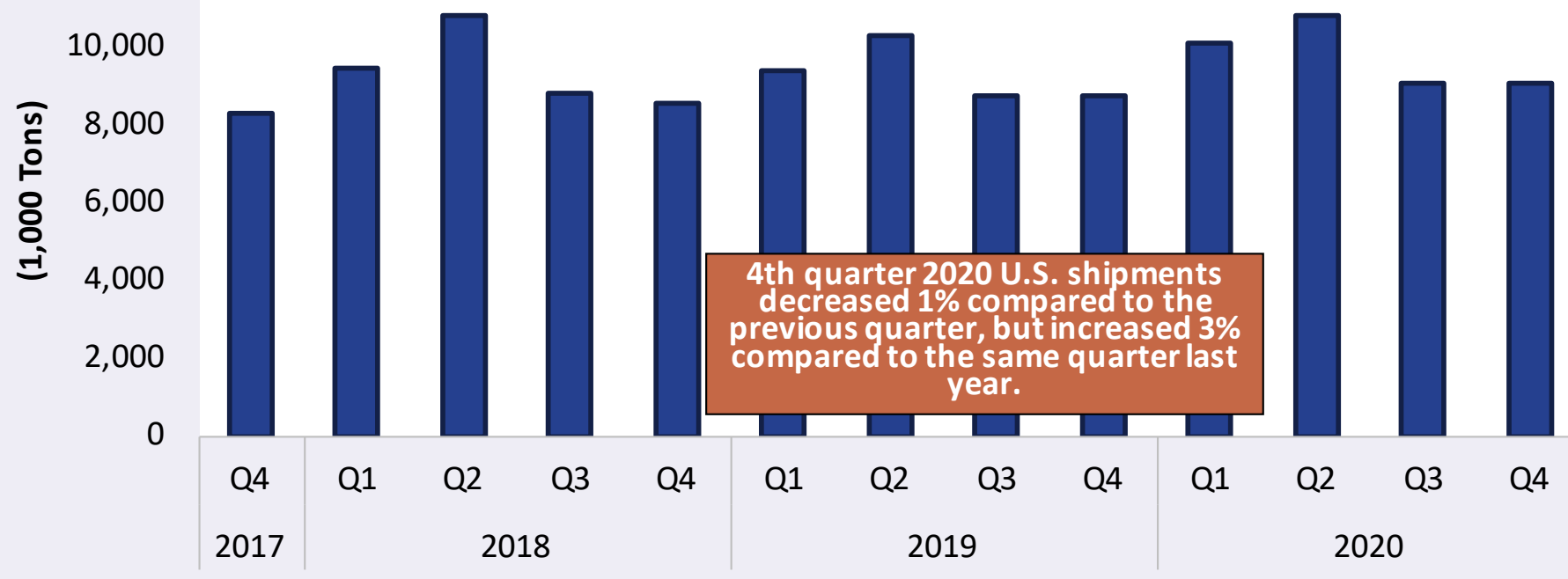

Source: AMS Transportation Services Division analysis of AMS Specialty Crops Program Market News data.

Table 8: Reported U.S. Fruit and Vegetable Shipments (1,000 Tons)

\begin{tabular}{|c|c|c|c|c|c|}
\hline Year & 1st Quarter & 2nd Quarter & 3rd Quarter & 4th Quarter & Annual \\
\hline 2020 & 10,081 & 10,750 & 9,060 & 9,009 & 38,899 \\
\hline 2019 & 9,381 & 10,246 & 8,687 & 8,714 & 37,028 \\
\hline 2018 & 9,421 & 10,795 & 8,789 & 8,503 & 37,508 \\
\hline 2017 & 8,072 & 9,642 & 8,479 & 8,267 & 34,459 \\
\hline 2016 & 8,094 & 9,761 & 8,541 & 8,188 & 34,583 \\
\hline 2015 & 8,118 & 9,630 & 8,324 & 7,771 & 33,842 \\
\hline 2014 & 7,733 & 9,139 & 8,080 & 7,725 & 32,677 \\
\hline 2013 & 7,451 & 8,972 & 7,762 & 7,527 & 31,712 \\
\hline 2012 & 7,577 & 9,008 & 7,774 & 7,532 & 31,890 \\
\hline 2011 & 7,007 & 8,981 & 7,887 & 7,988 & 31,863 \\
\hline 2010 & 7,065 & 8,881 & 7,985 & 7,522 & 31,454 \\
\hline 2009 & 7,158 & 8,728 & 7,990 & 7,270 & 31,147 \\
\hline 2008 & 7,059 & 8,666 & 7,426 & 6,904 & 30,057 \\
\hline 2007 & 6,959 & 8,585 & 7,475 & 7,099 & 30,118 \\
\hline 2006 & 6,335 & 8,400 & 7,854 & 6,960 & 29,550 \\
\hline 2005 & 6,877 & 8,324 & 7,737 & 7,387 & 30,325 \\
\hline 2004 & 6,867 & 8,331 & 6,876 & 6,732 & 28,807 \\
\hline
\end{tabular}

Source: AMS Transportation Services Division analysis of AMS Specialty Crops Program Market News data.

Note: Table values may not conform exactly due to rounding. 


\section{Reported Shipments by Selected Commodities}

Table 9: Reported Top 10 Commodity Shipments (1,000 Tons)

\begin{tabular}{|r|c|c|c|c|c|}
\hline Commodity & 4th Quarter & Previous & Same Quarter & \multicolumn{2}{|c|}{\begin{tabular}{c} 
Current Quarter as \% change from: \\
\cline { 5 - 6 }
\end{tabular}} \\
& $\mathbf{2 0 2 0}$ & Quarter & Last Year & Previous Qtr & Same Qtr Last Year \\
\hline Potatoes & 1,166 & 1,082 & 1,090 & $8 \%$ & $7 \%$ \\
\hline Apples & 882 & 707 & 903 & $25 \%$ & $-2 \%$ \\
\hline Onions Dry & 577 & 611 & 552 & $-5 \%$ & $5 \%$ \\
\hline Oranges & 437 & 211 & 486 & $108 \%$ & $-10 \%$ \\
\hline Tomatoes & 380 & 495 & 499 & $-23 \%$ & $-24 \%$ \\
\hline Grapes & 361 & 383 & 345 & $-6 \%$ & $5 \%$ \\
\hline Avocados & 339 & 532 & 592 & $-36 \%$ & $-43 \%$ \\
\hline Cucumbers & 295 & 316 & 451 & $-7 \%$ & $-35 \%$ \\
\hline Lettuce, Iceberg & 289 & 345 & 353 & $-16 \%$ & $-18 \%$ \\
\hline Celery & 255 & 200 & 254 & $27 \%$ & $1 \%$ \\
\hline
\end{tabular}

Source: AMS Transportation Services Division analysis of AMS Specialty Crops Program Market News data.

Note: Table values may not conform exactly due to rounding.

Table 10: Reported Top 10 Regions (1,000 Tons)

\begin{tabular}{|r|c|c|c|}
\hline \multicolumn{1}{|c|}{ Origin } & 4th Quarter 2020 Volume & \% Change from Last Quarter & $\begin{array}{c}\text { \% Change From Same Quarter } \\
\text { Last Year }\end{array}$ \\
\hline Mexico & 2,676 & $42 \%$ & $15 \%$ \\
\hline California & 2,339 & $-28 \%$ & $-4 \%$ \\
\hline PNW & 1,841 & $11 \%$ & $-2 \%$ \\
\hline Arizona & 386 & $2,054 \%$ & $-9 \%$ \\
\hline Florida & 305 & $1,340 \%$ & $-21 \%$ \\
\hline Great Lakes & 284 & $-24 \%$ & $7 \%$ \\
\hline Colorado & 248 & $86 \%$ & $11 \%$ \\
\hline Southeast & 180 & $-58 \%$ & $-11 \%$ \\
\hline Canada & 180 & $-17 \%$ & $706 \%$ \\
\hline New York & 141 & $68 \%$ & $-5 \%$ \\
\hline
\end{tabular}

Source: AMS Transportation Services Division analysis of AMS Specialty Crops Program Market News data.

Note: Table values may not conform exactly due to rounding.

Note: On Jan. 1, 2020, Specialty Crops Market News transitioned to the Department of Homeland Security ACE reporting system. According to AMS Speciality Crops Program, ACE is a more complete data system than their prior source and is an important factor behind the large increase in movements from the Canada border. 


\section{REgional MARKETS \\ U.S.-Mexico Border}

\section{Volume}

Total reported shipments of fruits and vegetables from Mexico during fourth quarter 2020 were 2.7 million tons, a 15-percent increase year to year. The sum of the top five commodities also increased 15 percent year to year. Each of the top five commodities increased, with particularly sharp increases of tomatoes (35 percent) and cucumbers (15 percent).

\section{Rates}

In fourth quarter 2020, truck rates for shipments between 501 miles and 1,500 miles from the Arizona border averaged $\$ 2.81$ per mile, up 30 percent from quarter to quarter and up 27 percent year to year. Rates for shipments between 501 miles and 1,500 miles from the Texas border averaged \$2.88 per mile, up 22 percent quarter to quarter and up 29 percent year to year.

\section{Truck Overview}

Diesel fuel prices for border crossings from Arizona averaged \$2.65 per gallon, increasing by 3 percent quarter to quarter but decreasing by 21 percent year to year. Diesel fuel prices for border crossings from Texas averaged \$2.22 per gallon, a 2-percent increase quarter to quarter, but a 20-percent decrease year to year. Shippers at the Arizona-Mexico border and the Texas-Mexico border both reported adequate availability in October and slight shortage in November and December.

Table 11: Reported Top Five Commodities Shipped from Mexico (1,000 tons)

\begin{tabular}{|c|c|c|c|c|c|c|}
\hline \multirow[t]{2}{*}{ Commodity } & \multirow{2}{*}{$\begin{array}{c}\text { 4th Quarter } \\
2020\end{array}$} & \multirow{2}{*}{$\begin{array}{l}\text { Share of } \\
\text { Mexico- } \\
\text { Total Total }\end{array}$} & \multirow{2}{*}{$\begin{array}{l}\text { Previous } \\
\text { Quarter }\end{array}$} & \multirow{2}{*}{$\begin{array}{c}\text { Same } \\
\text { Quarter } \\
\text { Last Year }\end{array}$} & \multicolumn{2}{|c|}{$\begin{array}{c}\text { Current Quarter as \% change } \\
\text { from: }\end{array}$} \\
\hline & & & & & Previous Qtr & Same Qtr Last Year \\
\hline Avocados & 320 & $12 \%$ & 227 & 292 & $41 \%$ & $9 \%$ \\
\hline Cucumbers & 237 & $9 \%$ & 104 & 205 & $128 \%$ & $15 \%$ \\
\hline Tomatoes & 227 & $8 \%$ & 153 & 168 & $48 \%$ & $35 \%$ \\
\hline Squash & 170 & $6 \%$ & 30 & 157 & $464 \%$ & $8 \%$ \\
\hline Peppers, Other & 169 & $6 \%$ & 132 & 156 & $28 \%$ & $8 \%$ \\
\hline Top 5 Total & 1,122 & $42 \%$ & 646 & 979 & $74 \%$ & $15 \%$ \\
\hline Mexico Total & 2,676 & $100 \%$ & 1,880 & 2,332 & $42 \%$ & $15 \%$ \\
\hline
\end{tabular}

Source: AMS Transportation Services Division analysis of AMS Specialty Crops Program Market News data.

Any "-" in the table indicates no reported shipments during the quarter or a percentage change with an at- or near-zero denominator. Note: Table values may not conform exactly due to rounding. 
Table 12: Top 5 Commodities Shipped to U.S. from Mexico by State of Entry (1,000 tons)

\begin{tabular}{|c|c|c|c|c|c|c|c|}
\hline \multicolumn{2}{|l|}{ Texas } & \multicolumn{2}{|c|}{ California } & \multicolumn{2}{|l|}{ Arizona } & \multicolumn{2}{|c|}{ New Mexico } \\
\hline Commodity & $\begin{array}{c}\text { 4th } \\
\text { Quarter } \\
2020\end{array}$ & Commodity & $\begin{array}{c}\text { 4th } \\
\text { Quarter } \\
2020\end{array}$ & Commodity & $\begin{array}{c}\text { 4th } \\
\text { Quarter } \\
2020\end{array}$ & Commodity & $\begin{array}{c}\text { 4th } \\
\text { Quarter } \\
2020\end{array}$ \\
\hline Avocados & 314 & $\begin{array}{c}\text { Tomatoes, Plum } \\
\text { Type }\end{array}$ & 49 & Cucumbers & 153 & Peppers, Other & 81 \\
\hline Tomatoes & 181 & Onions Green & 43 & Squash & 149 & Corn-Sweet & $<1$ \\
\hline Limes & 138 & Misc Tropical & 35 & $\begin{array}{l}\text { Watermelons, } \\
\text { Seedless }\end{array}$ & 133 & Misc Tropical & $<1$ \\
\hline $\begin{array}{l}\text { Tomatoes, } \\
\text { Plum Type }\end{array}$ & 95 & Peppers, Other & 20 & $\begin{array}{c}\text { Peppers, Bell } \\
\text { Type }\end{array}$ & 53 & Watermelons & $<1$ \\
\hline Broccoli & 79 & Asparagus & 18 & Tomatoes & 35 & $\begin{array}{c}\text { Cactus Leaf } \\
\text { (Nopales) }\end{array}$ & $<1$ \\
\hline $\begin{array}{c}\text { Mexico through } \\
\text { TX Total }\end{array}$ & 1,508 & $\begin{array}{c}\text { Mexico through } \\
\text { CA Total }\end{array}$ & 344 & $\begin{array}{c}\text { Mexico through } \\
\text { AZ Total }\end{array}$ & 741 & $\begin{array}{c}\text { Mexico through } \\
\text { NM Total }\end{array}$ & 82 \\
\hline
\end{tabular}

Source: AMS Transportation Services Division analysis of AMS Specialty Crops Program Market News data.

Any "-" in the table indicates no reported shipments during the quarter.

Note: Table values may not conform exactly due to rounding.

Figure 5: Truck Rates from U.S.-Mexico Border (\$/Mile by Distance Traveled)

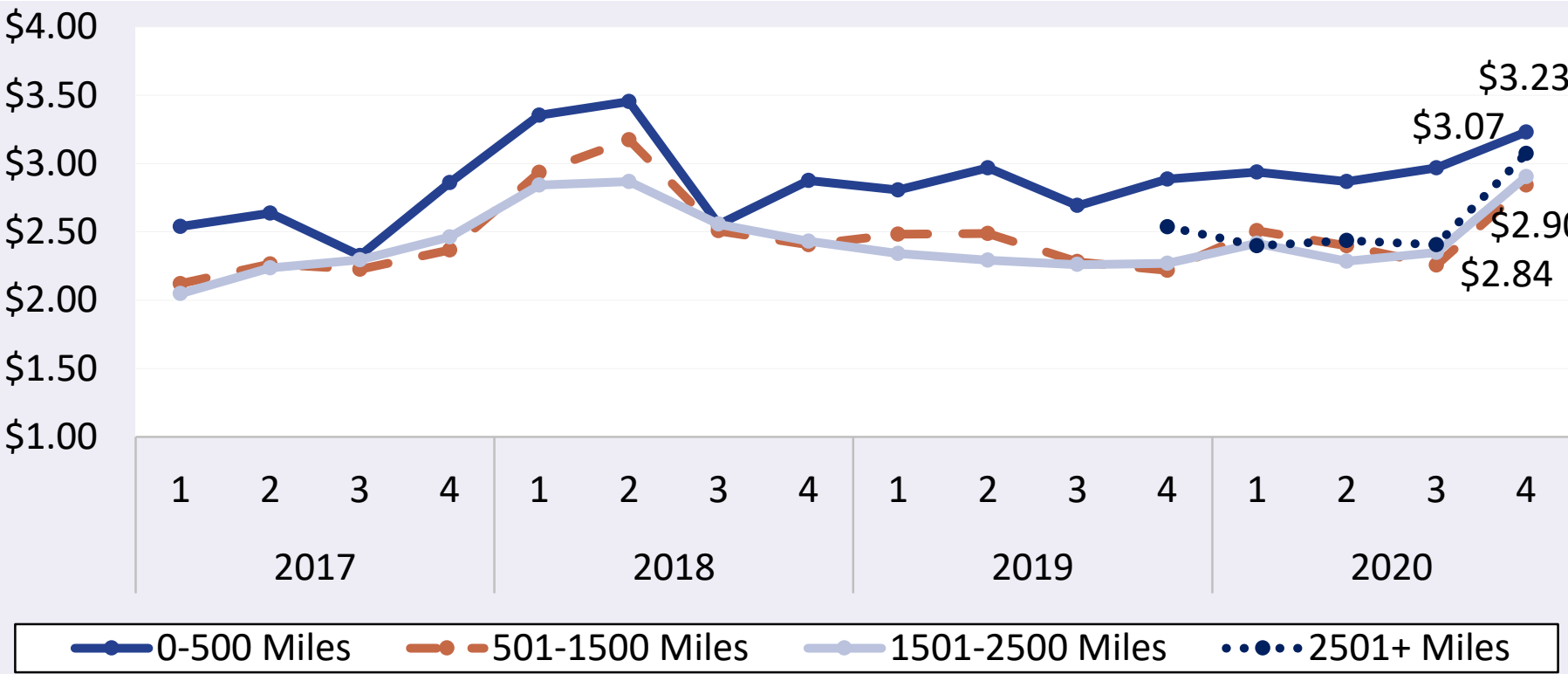

Source: AMS Transportation Services Division analysis of AMS Specialty Crops Program Market News data.

Any gaps in the chart lines are the result of quarters with no reported data for the region. 


\section{Agricultural Refrigerated Truck Quarterly \\ Quarter 4, 2020}

Figure 6: Texas Truck Rates from U.S.-Mexico Border (\$/Mile by Distance Traveled) $\$ 4.00$

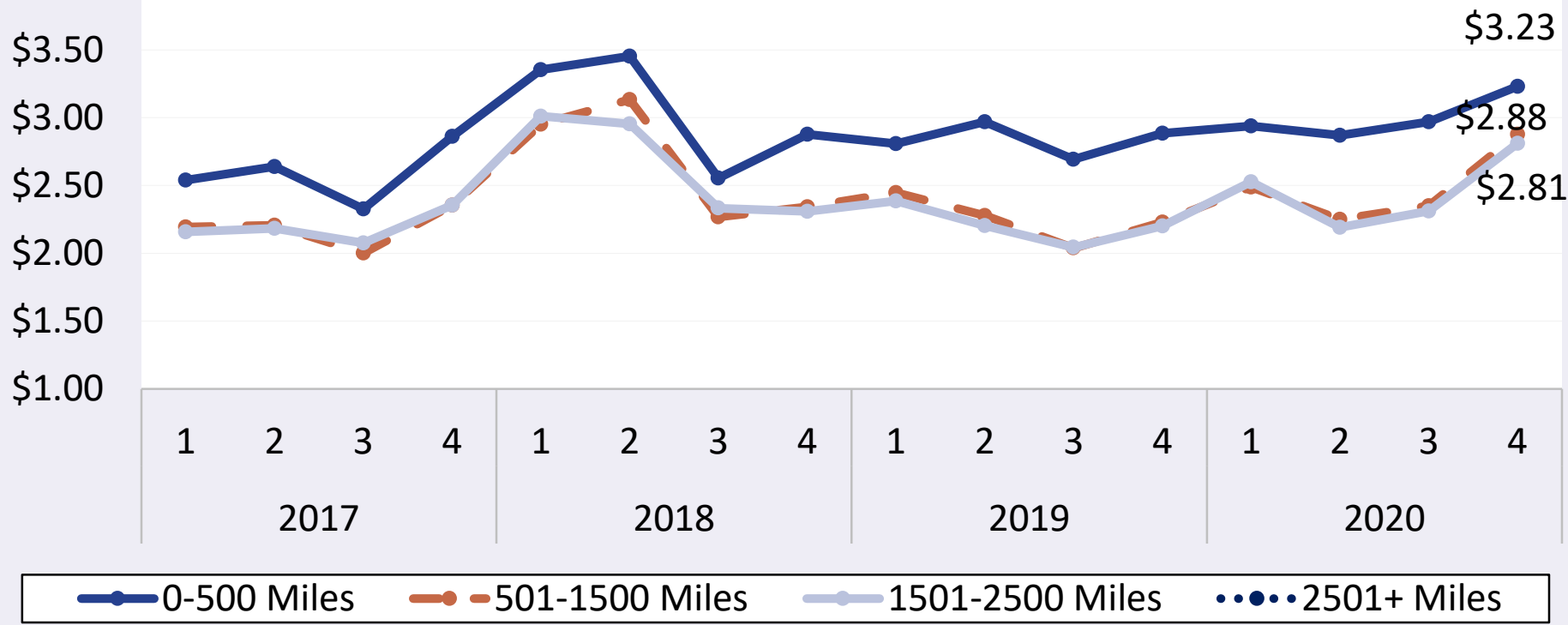

Source: AMS Transportation Services Division analysis of AMS Specialty Crops Program Market News data. Any gaps in the chart lines are the result of quarters with no reported data for the region.

Figure 7: Arizona Truck Rates from U.S.-Mexico Border (\$/Mile by Distance Traveled)

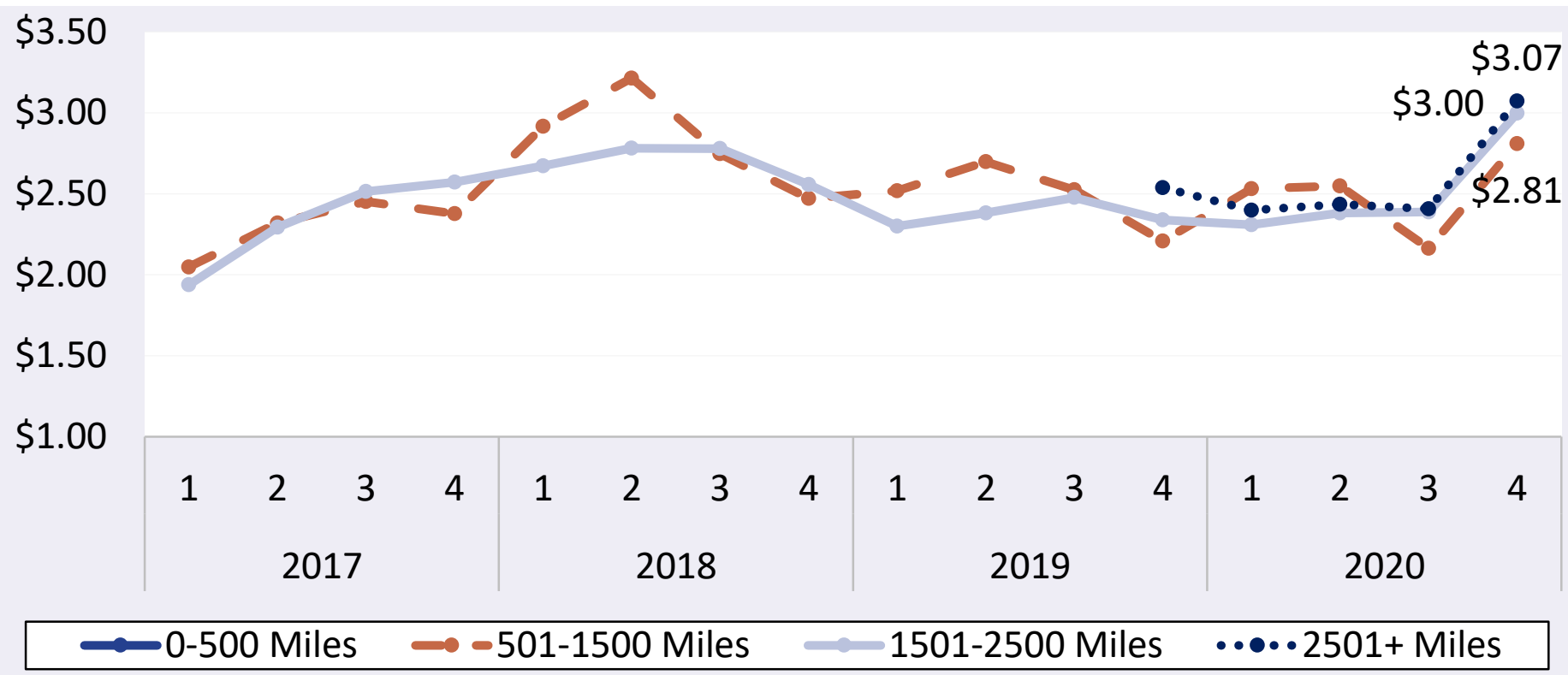

Source: AMS Transportation Services Division analysis of AMS Specialty Crops Program Market News data. Any gaps in the chart lines are the result of quarters with no reported data for the region. 


\section{Agricultural Refrigerated Truck Quarterly \\ Quarter 4, 2020}

Table 13: Truck Overview from the U.S.-Mexico Border

\begin{tabular}{|c|c|c|c|c|}
\hline Region/Reporting District & October & November & December & 4th Quarter \\
\hline Mexico Crossings Through Nogales, Arizona & 3.47 & 4.5 & 4 & 3.99 \\
\hline Mexico Crossings Through Texas & 3.25 & 4 & 4.2 & 3.82 \\
\hline Regional Average Availability & 3.36 & 4.25 & 4.1 & 3.90 \\
\hline Diesel Fuel Price (\$/gallon), through Texas & 2.15 & 2.18 & 2.34 & 2.22 \\
\hline Diesel Fuel Price (\$/gallon), through Arizona & 2.54 & 2.65 & 2.75 & 2.65 \\
\hline
\end{tabular}

Source: AMS Transportation Services Division analysis of AMS Specialty Crops Program Market News data.

Any "-" in the table indicates no reported shipments during the quarter.

Note: Table values may not conform exactly due to rounding.

Note: AMS Specialty Crops Program defines regions by commodity, which may overlap in truck availability reporting.

\section{Figure 8: Refrigerated Truck Availability Monthly Ratings from the U.S-Mexico Border}

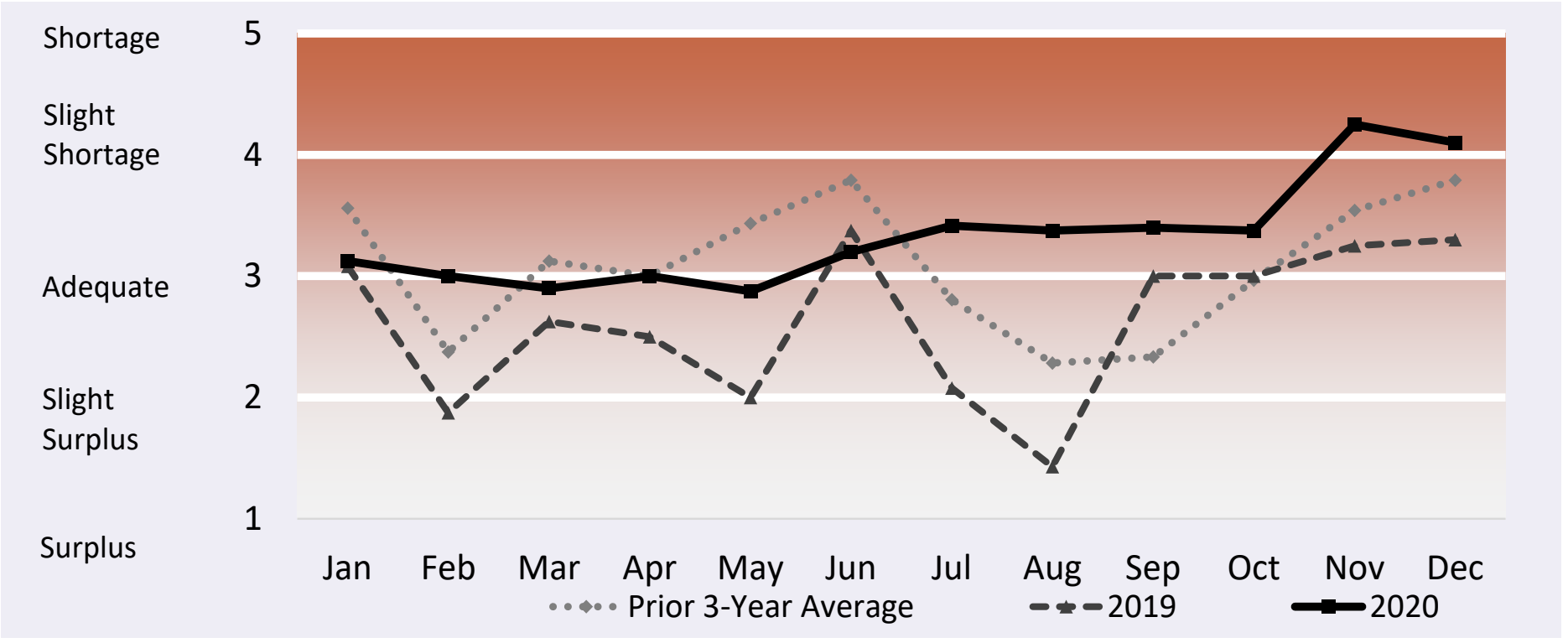

Source: AMS Transportation Services Division analysis of AMS Specialty Crops Program Market News data. Any gaps in the chart lines are the result of quarters with no reported data for the region. 


\section{California}

\section{Volume}

Total reported shipments of fruits and vegetables from California during fourth quarter 2020 were 2.34 million tons, a decrease of 4 percent from the same quarter last year (year to year). The top five commodities decreased by 1 percent from year to year. Two of the top 5 commodities saw year-to-year decreases this quarter, including oranges (12 percent) and lemons (12 percent). Shipments of celery increased by 16 percent, grapes by 5 percent, and clementines by 4 percent.

\section{Rates}

The quarterly average truck rate for shipments between 501 miles and 1,500 miles was $\$ 3.87$ per mile, a 27-percent increase quarter to quarter, and a 28-percent increase year to year.

\section{Truck Overview}

Diesel fuel prices averaged \$3.28 per gallon, a 1-percent increase quarter to quarter but a 17-percent decrease year to year. All districts in California reported adequate truck availability in October. In November San Joaquin Valley reported adequate availability while all other districts reported a slight truck shortage. In December, Oxnard District reported a slight truck shortage while all other districts reported adequate truck availability.

Table 14: Reported Top Five Commodities Shipped from California (1,000 tons)

\begin{tabular}{|r|c|c|c|c|c|c|}
\hline \multirow{2}{*}{ Commodity } & $\begin{array}{c}\text { 4th Quarter } \\
2020\end{array}$ & $\begin{array}{c}\text { Share of } \\
\text { California } \\
\text { Total }\end{array}$ & $\begin{array}{c}\text { Previous } \\
\text { Quarter }\end{array}$ & $\begin{array}{c}\text { Same } \\
\text { Quarter } \\
\text { Last Year }\end{array}$ & \multicolumn{2}{|c|}{$\begin{array}{c}\text { Current Quarter as } \% \text { change } \\
\text { from: }\end{array}$} \\
\cline { 6 - 8 } Oranges & 369 & $16 \%$ & 205 & 417 & $80 \%$ & $-12 \%$ \\
\hline Grapes & 361 & $15 \%$ & 364 & 345 & $-1 \%$ & $5 \%$ \\
\hline Clementines & 240 & $10 \%$ & - & 231 & - & $4 \%$ \\
\hline Celery & 233 & $10 \%$ & 171 & 201 & $36 \%$ & $16 \%$ \\
\hline Lemons & 179 & $8 \%$ & 138 & 204 & $30 \%$ & $-12 \%$ \\
\hline Top 5 Total & 1,382 & $59 \%$ & 877 & 1,398 & $58 \%$ & $-1 \%$ \\
\hline California Total & 2,339 & $100 \%$ & 3,258 & 2,439 & $-28 \%$ & $-4 \%$ \\
\hline
\end{tabular}

Source: AMS Transportation Services Division analysis of AMS Specialty Crops Program Market News data.

Any "-" in the table indicates no reported shipments during the quarter or a percentage change with an at- or near-zero denominator. Note: Table values may not conform exactly due to rounding. 
Figure 9: California Truck Rates (\$/Mile by Distance Travelled)

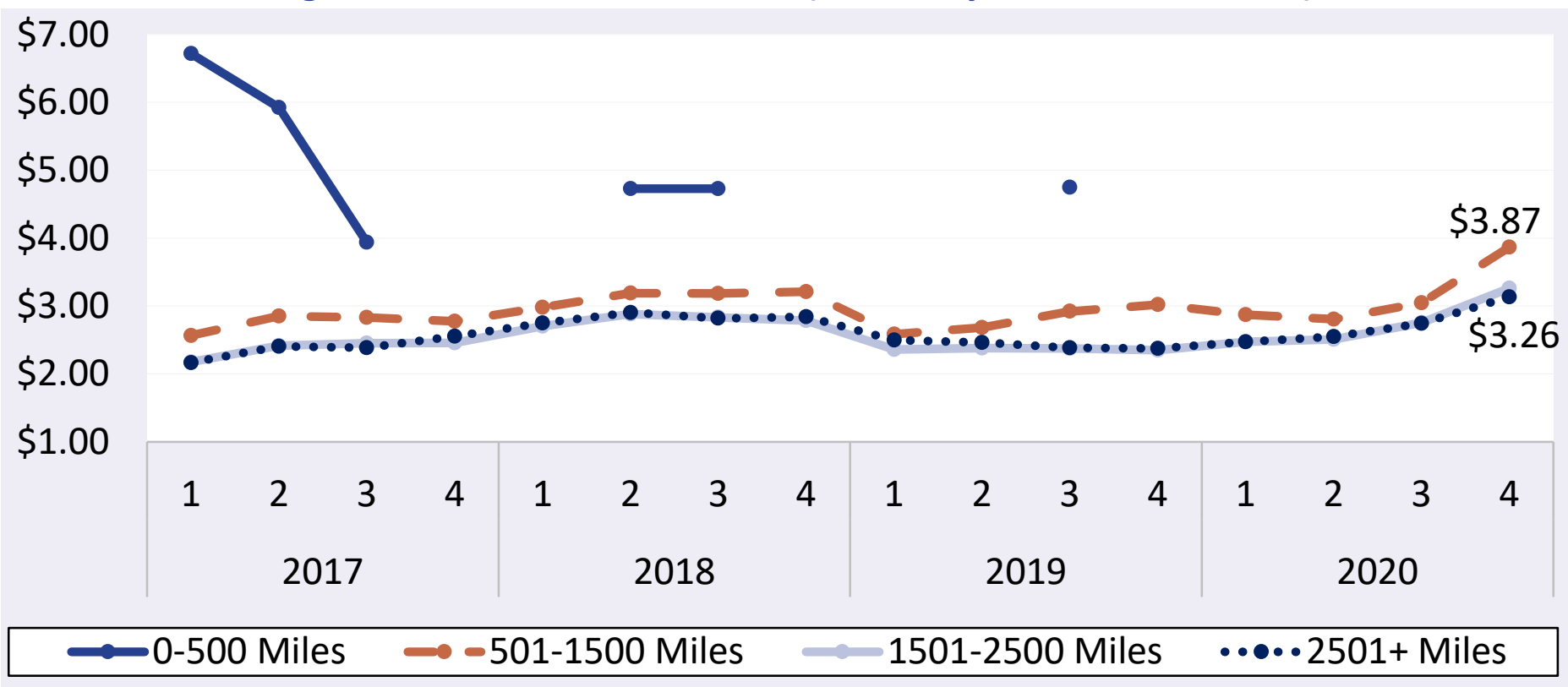

Source: AMS Transportation Services Division analysis of AMS Specialty Crops Program Market News data. Any gaps in the chart lines are the result of quarters with no reported data for the region.

Table 15: California Truck Overview (Availability Rating: 1=Surplus to 5=Shortage)

\begin{tabular}{|r|c|c|c|c|}
\hline \multicolumn{1}{|c|}{ Region/Reporting District } & October & November & December & 4th Quarter \\
\hline $\begin{array}{r}\text { Imperial, Palo Verde And Coachella Valleys, } \\
\text { California And Central And Western Arizona }\end{array}$ & - & - & 3.33 & 3.33 \\
\hline Kern District California & 3 & 4 & 3.8 & 3.6 \\
\hline Oxnard District California & - & 4.67 & 3.8 & 4.23 \\
\hline Salinas-Watsonville California & 3 & 4.25 & 4.5 & 3.92 \\
\hline San Joaquin Valley California & 3 & 3.5 & 3.4 & 3.3 \\
\hline Santa Maria California & 3 & 4.25 & 3.8 & 3.68 \\
\hline South District California & 3 & 4 & 3.6 & 3.53 \\
\hline Regional Average Availability & 3 & 4.11 & 3.75 & 3.62 \\
\hline Diesel Fuel Price (\$/gallon) & 3.25 & 3.25 & 3.36 & 3.28 \\
\hline
\end{tabular}

Source: AMS Transportation Services Division analysis of AMS Specialty Crops Program Market News data.

Any "-" in the table indicates no reported shipments during the quarter.

Note: Table values may not conform exactly due to rounding.

Note: AMS Specialty Crops Program defines regions by commodity, which may overlap in truck availability reporting. 


\section{Agricultural Refrigerated Truck Quarterly \\ Quarter 4, 2020}

Figure 10: Refrigerated Truck Availability Monthly Ratings for California

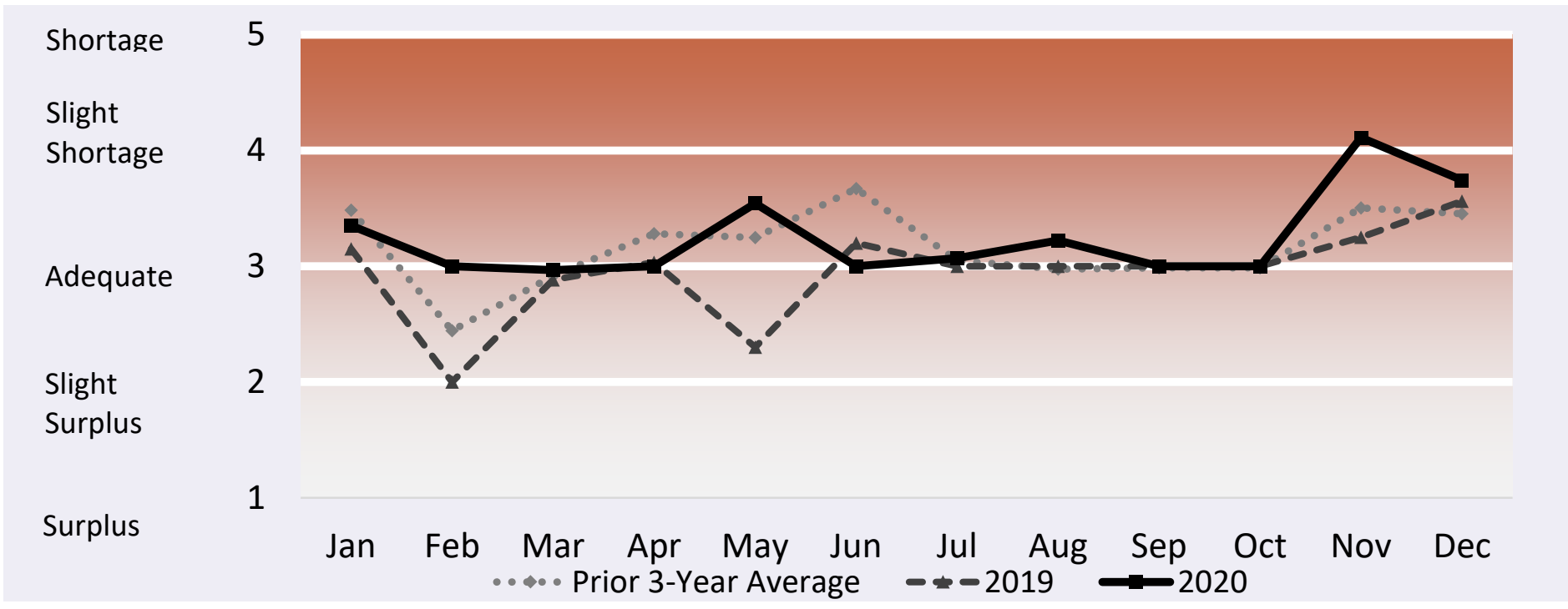

Source: AMS Transportation Services Division analysis of AMS Specialty Crops Program Market News data.

Any gaps in the chart lines are the result of quarters with no reported data for the region. 


\section{PNW}

\section{Volume}

During fourth quarter 2020, total reported shipments of fruits and vegetables from the PNW were 1.84 million tons, decreasing by 2 percent year to year. The sum of the top five commodities also decreased by 2 percent year to year. Except for dry onions (3 percent increase), the rest of the top five commodities saw year-to-year decreases this quarter: cranberries ( 23 percent), pears ( 7 percent), apples ( 4 percent), and potatoes (1 percent).

\section{Rates}

The quarterly average truck rate for shipments between 501 miles and 1,500 miles was $\$ 2.37$ per mile, increasing 16 percent quarter to quarter, and 8 percent year to year.

\section{Truck Overview}

Diesel fuel prices averaged $\$ 2.65$ per gallon, with an increase of 3 percent quarter to quarter but a 21 percent decrease year to year. Shippers in all districts except the Yakima Valley and Wenatchee District of Washington reported shortage of truck availability in October, all districts reported shortage in November, and in December Yakima Valley and Wenatchee District reported adequate truck availability while all other districts reported a slight shortage.

Table 16: Reported Top Five Commodities Shipped from PNW (1,000 tons)

\begin{tabular}{|c|c|c|c|c|c|c|}
\hline \multirow[t]{2}{*}{ Commodity } & \multirow{2}{*}{$\begin{array}{l}\text { 4th Quarter } \\
2020\end{array}$} & \multirow{2}{*}{$\begin{array}{l}\text { Share of } \\
\text { PNW Total }\end{array}$} & \multirow{2}{*}{$\begin{array}{l}\text { Previous } \\
\text { Quarter }\end{array}$} & \multirow{2}{*}{$\begin{array}{c}\text { Same } \\
\text { Quarter } \\
\text { Last Year }\end{array}$} & \multicolumn{2}{|c|}{$\begin{array}{l}\text { Current Quarter as \% change } \\
\text { from: }\end{array}$} \\
\hline & & & & & Previous Qtr & Same Qtr Last Yeal \\
\hline Apples & 714 & $39 \%$ & 652 & 741 & $9 \%$ & $-4 \%$ \\
\hline Potatoes & 527 & $29 \%$ & 534 & 531 & $-1 \%$ & $-1 \%$ \\
\hline Onions Dry & 445 & $24 \%$ & 282 & 431 & $58 \%$ & $3 \%$ \\
\hline Pears & 154 & $8 \%$ & 46 & 165 & $231 \%$ & $-7 \%$ \\
\hline Cranberries & 1 & $<1 \%$ & $<1$ & 2 & - & $-23 \%$ \\
\hline Top 5 Total & 1,840 & $100 \%$ & 1,515 & 1,871 & $21 \%$ & $-2 \%$ \\
\hline PNW Total & 1,841 & $100 \%$ & 1,659 & 1,871 & $11 \%$ & $-2 \%$ \\
\hline
\end{tabular}

Source: AMS Transportation Services Division analysis of AMS Specialty Crops Program Market News data.

Any "-" in the table indicates no reported shipments during the quarter or a percentage change with an at- or near-zero denominator. Note: Table values may not conform exactly due to rounding. 
Figure 11: PNW Truck Rates (\$/Mile by Distance Travelled)

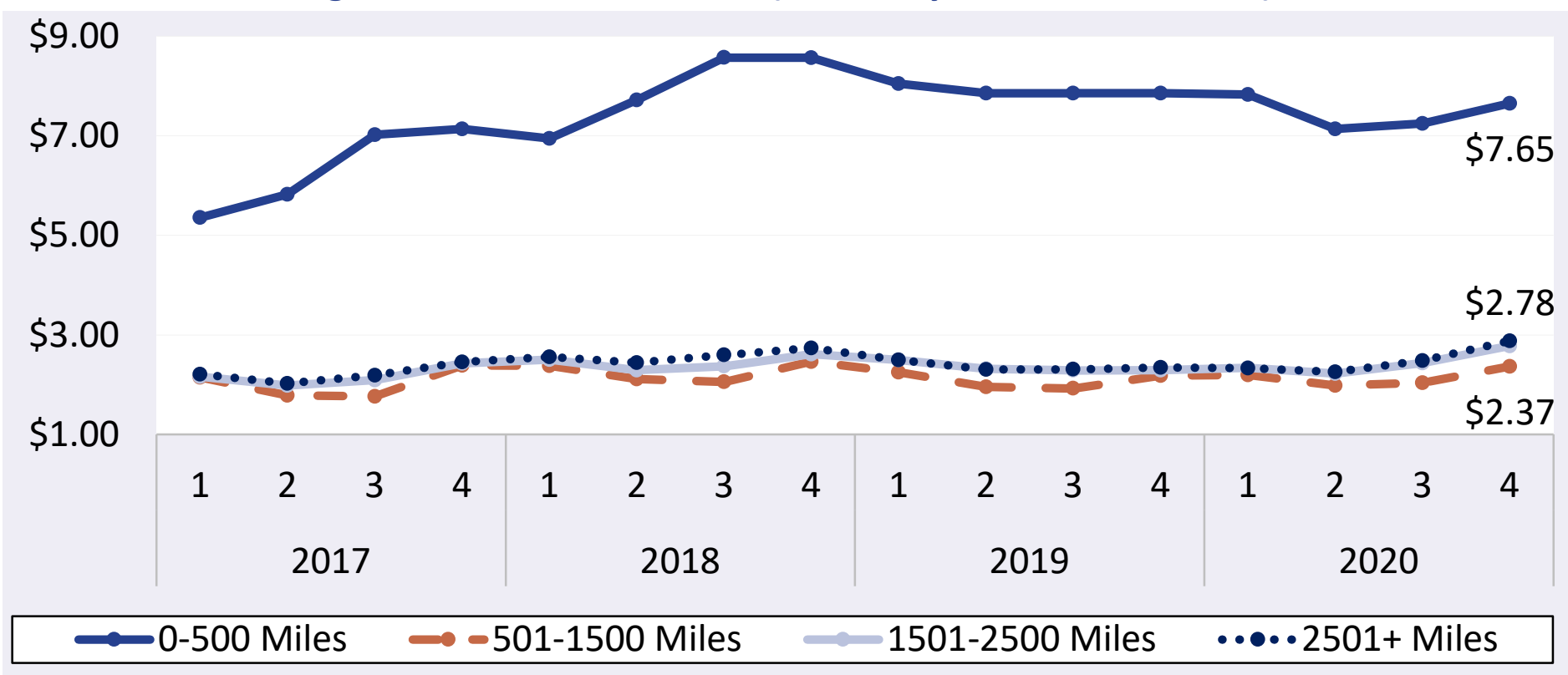

Source: AMS Transportation Services Division analysis of AMS Specialty Crops Program Market News data. Any gaps in the chart lines are the result of quarters with no reported data for the region.

Table 17: PNW Truck Overview (Availability Rating: 1=Surplus to 5=Shortage)

\begin{tabular}{|c|c|c|c|c|}
\hline Region/Reporting District & October & November & December & 4th Quarter \\
\hline Columbia Basin Washington & 5 & 5 & 4.4 & 4.8 \\
\hline Idaho And Malheur County, Oregon & 5 & 5 & 4.4 & 4.8 \\
\hline $\begin{array}{c}\text { Upper Valley, Twin Falls-Burley District Idaho } \\
\text { Yakima Valley \& Wenatchee District } \\
\text { Washington }\end{array}$ & 5.25 & 5 & 4.2 & 4.73 \\
\hline $\begin{array}{c}\text { Regional Average Availability } \\
\text { Diesel Fuel Price (\$/gallon) }\end{array}$ & $\mathbf{4 . 5 6}$ & $\mathbf{2}$ & $\mathbf{3}$ & 3.88 \\
\hline
\end{tabular}

Source: AMS Transportation Services Division analysis of AMS Specialty Crops Program Market News data. Any "-" in the table indicates no reported shipments during the quarter.

Note: Table values may not conform exactly due to rounding.

Note: AMS Specialty Crops Program defines regions by commodity, which may overlap in truck availability reporting. 


\section{Agricultural Refrigerated Truck Quarterly \\ Quarter 4, 2020}

Figure 12: Refrigerated Truck Availability Monthly Ratings for PNW

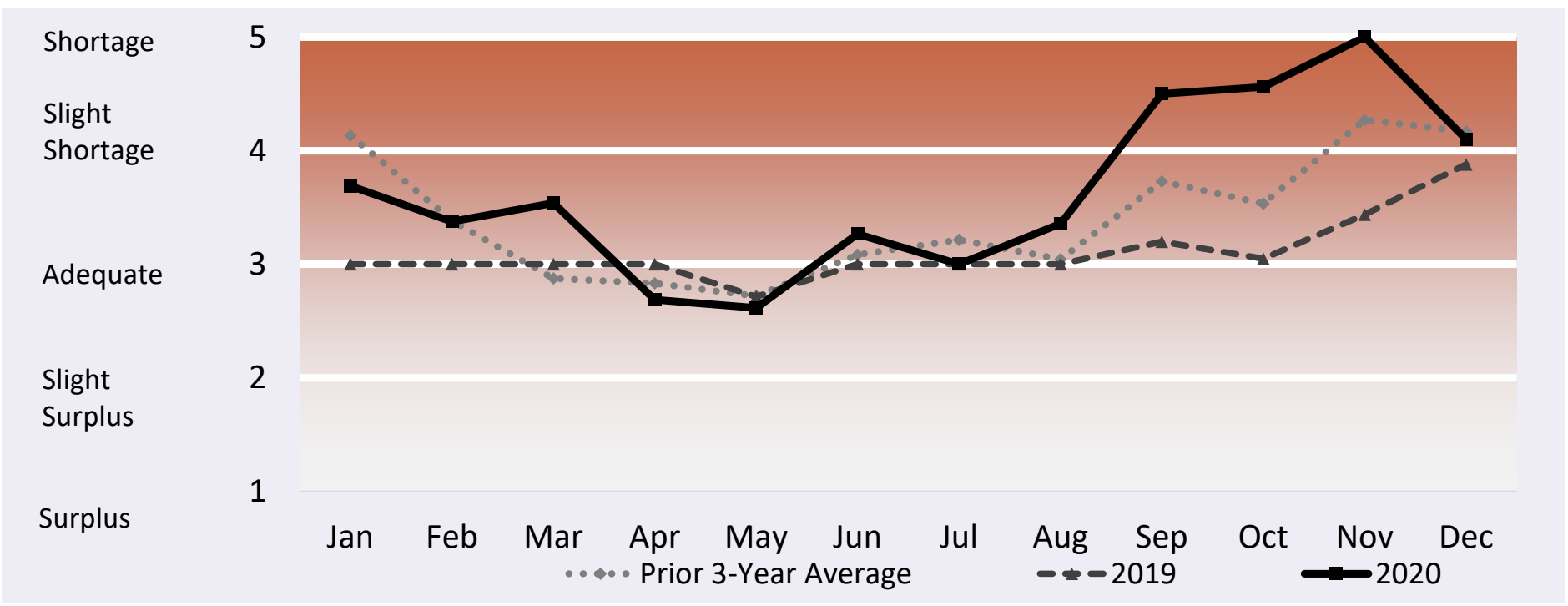

Source: AMS Transportation Services Division analysis of AMS Specialty Crops Program Market News data. Any gaps in the chart lines are the result of quarters with no reported data for the region. 


\section{Arizona}

\section{Volume}

During fourth quarter 2020, total reported shipments of fruits and vegetables from Arizona were 386,000 tons, down 9 percent year to year. The sum of the top five commodities also decreased 5 percent year to year. Decreases in broccoli (20 percent), iceberg lettuce (18 percent) and romaine lettuce ( 9 percent) offset strong increases in cantaloupe (54 percent) and moderate increases in processed lettuce (11 percent).

\section{Rates}

The quarterly average truck rate for shipments between 501 miles and 1,500 miles was $\$ 4.09$ per mile, up 29 percent quarter to quarter and up 37 percent year to year.

\section{Truck Overview}

Diesel fuel prices averaged $\$ 2.65$ per gallon, up 2 percent quarter to quarter but down 21 percent year to year. Data on truck availability were available only for the month of December for Imperial Palo Verde and Coachella Valleys, which reported adequate truck availability for that month.

Table 18: Reported Top Five Commodities Shipped from Arizona (1,000 tons)

\begin{tabular}{|r|c|c|c|c|c|c|}
\hline \multirow{2}{*}{ Commodity } & \multirow{4}{*}{$\begin{array}{c}\text { 4th Quarter } \\
2020\end{array}$} & $\begin{array}{c}\text { Share of } \\
\text { Arizona } \\
\text { Total }\end{array}$ & \multirow{2}{*}{$\begin{array}{c}\text { Previous } \\
\text { Quarter }\end{array}$} & $\begin{array}{c}\text { Same } \\
\text { Quarter } \\
\text { Last Year }\end{array}$ & \multicolumn{2}{c|}{$\begin{array}{c}\text { Current Quarter as } \% \text { change } \\
\text { from: }\end{array}$} \\
\cline { 6 - 7 } & & $27 \%$ & - & 128 & - & $-18 \%$ \\
\hline Lettuce, Iceberg & 105 & $27 \%$ & - & 114 & - & $-9 \%$ \\
\hline Lettuce, Promaine & 104 & $16 \%$ & - & 55 & - & $11 \%$ \\
\hline Cantaloupe & 42 & $11 \%$ & 6 & 27 & $651 \%$ & $54 \%$ \\
\hline Broccoli & 13 & $3 \%$ & - & 17 & - & $-20 \%$ \\
\hline Top 5 Total & 325 & $84 \%$ & 6 & 341 & $5,689 \%$ & $-5 \%$ \\
\hline Arizona Total & 386 & $100 \%$ & 18 & 422 & $2,054 \%$ & $-9 \%$ \\
\hline
\end{tabular}

Source: AMS Transportation Services Division analysis of AMS Specialty Crops Program Market News data.

Any "-" in the table indicates no reported shipments during the quarter or a percentage change with an at- or near-zero denominator. Note: Table values may not conform exactly due to rounding. 
Figure 13: Arizona Truck Rates (\$/Mile by Distance Travelled)

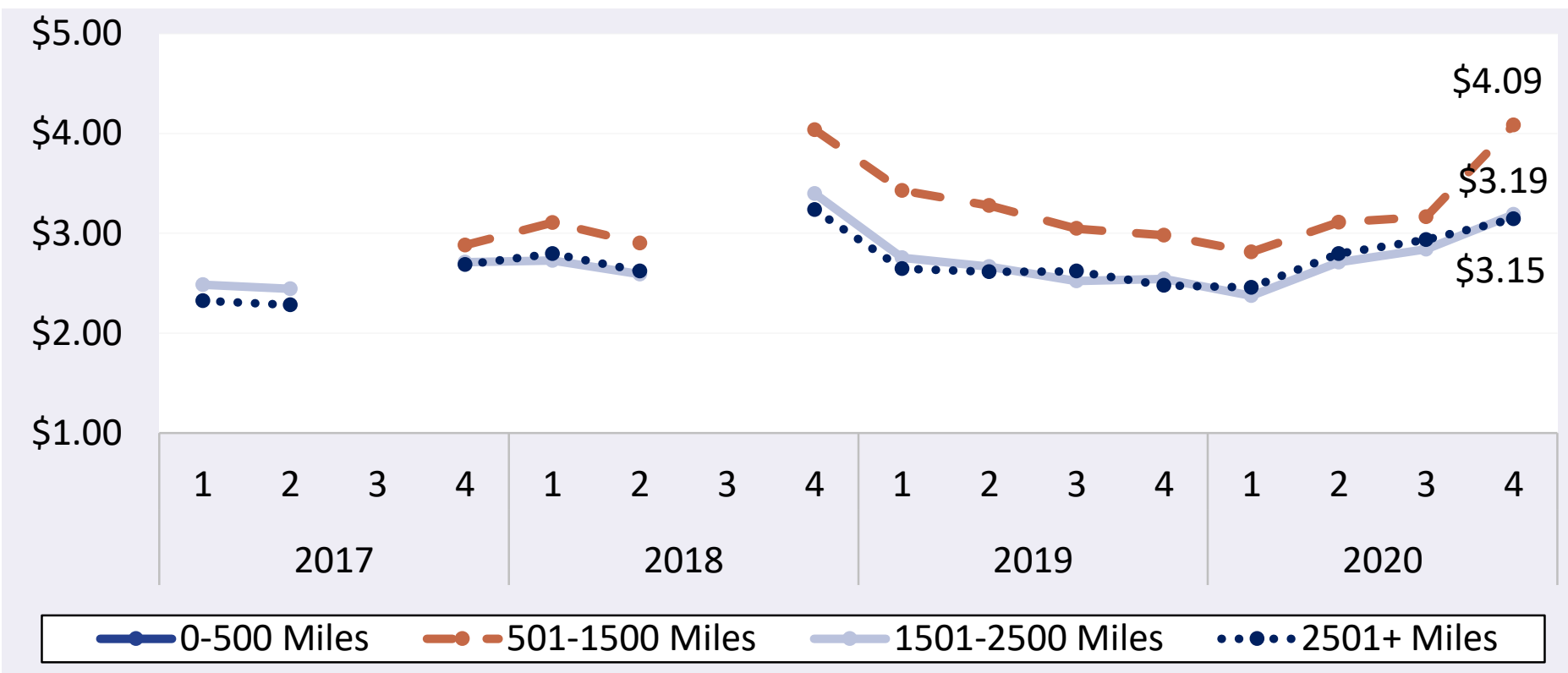

Source: AMS Transportation Services Division analysis of AMS Specialty Crops Program Market News data.

Any gaps in the chart lines are the result of quarters with no reported data for the region.

Table 19: Arizona Truck Overview (Availability Rating: 1=Surplus to 5=Shortage)

\begin{tabular}{|c|c|c|c|c|}
\hline Region/Reporting District & October & November & December & 4th Quarter \\
\hline $\begin{array}{c}\text { Imperial, Palo Verde And Coachella Valleys, } \\
\text { California And Central And Western Arizona }\end{array}$ & - & - & 3.33 & 3.33 \\
\hline Regional Average Availability & - & - & 3.33 & 3.33 \\
\hline Diesel Fuel Price (\$/gallon) & $\mathbf{2 . 5 4}$ & $\mathbf{2 . 6 5}$ & $\mathbf{2 . 7 5}$ & $\mathbf{2 . 6 5}$ \\
\hline
\end{tabular}

Source: AMS Transportation Services Division analysis of AMS Specialty Crops Program Market News data.

Any "-" in the table indicates no reported shipments during the quarter.

Note: Table values may not conform exactly due to rounding.

Note: AMS Specialty Crops Program defines regions by commodity, which may overlap in truck availability reporting. 


\section{Agricultural Refrigerated Truck Quarterly \\ Quarter 4, 2020}

Figure 14: Refrigerated Truck Availability Monthly Ratings for Arizona

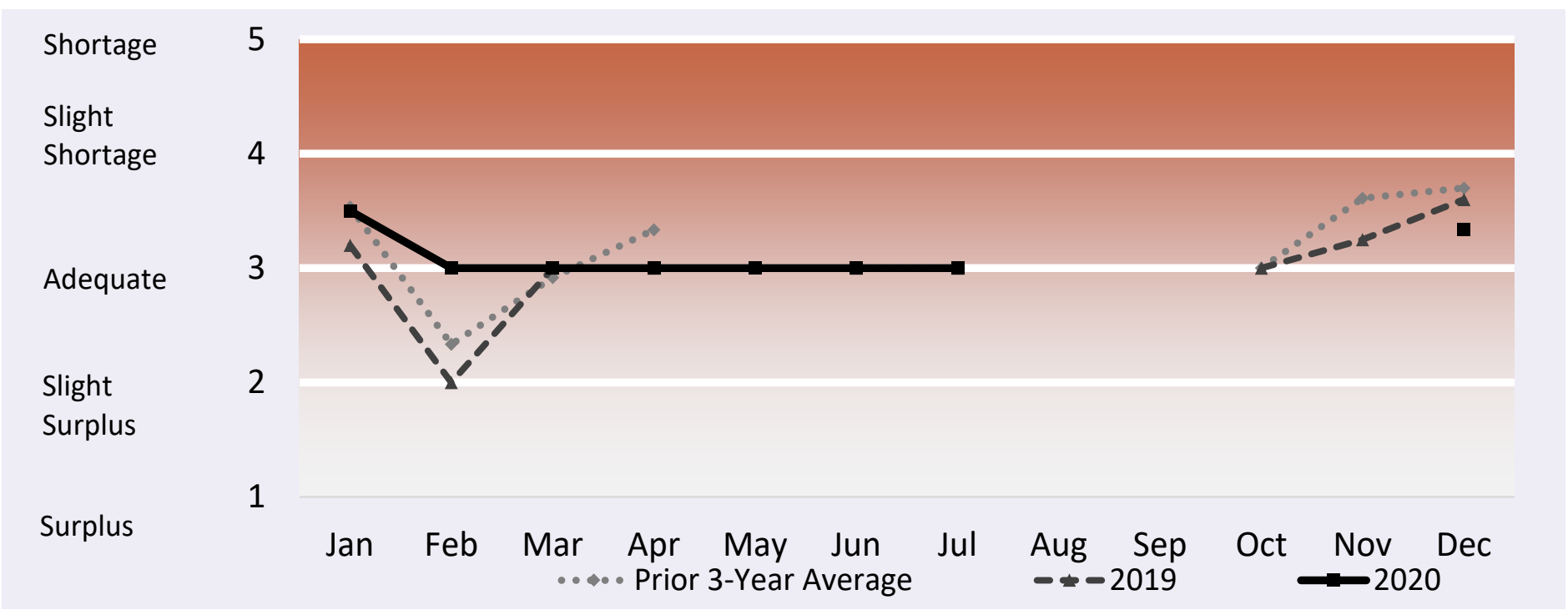

Source: AMS Transportation Services Division analysis of AMS Specialty Crops Program Market News data.

Any gaps in the chart lines are the result of quarters with no reported data for the region. 


\section{Florida}

\section{Volume}

Total reported shipments of fruits and vegetables from Florida during fourth quarter 2020 were 305,000 tons, down 21 percent year to year. The sum of the top five commodities decreased 13 percent year to year. Of the top five commodities, only grapefruit (4 percent) and oranges ( 3 percent) increased. Shipments decreased 23 percent for bell peppers, 22 percent for tomatoes, and 15 percent for plum tomatoes

\section{Rates}

The quarterly average truck rate for shipments between 501 miles and 1,500 miles was $\$ 2.36$ per mile, up 25 percent year to year. Data were not available for third quarter truck rates.

\section{Truck Overview}

Diesel fuel prices averaged $\$ 2.38$ per gallon, up 1 percent quarter to quarter but down 19 percent year to year. Shippers in Florida reported slight shortage in truck availability in the fourth quarter.

Table 20: Reported Top Five Commodities Shipped from Florida (1,000 tons)

\begin{tabular}{|c|c|c|c|c|c|c|}
\hline \multirow[t]{2}{*}{ Commodity } & \multirow{2}{*}{$\begin{array}{c}\text { 4th Quarter } \\
2020\end{array}$} & \multirow{2}{*}{$\begin{array}{c}\text { Share of } \\
\text { Florida } \\
\text { Total }\end{array}$} & \multirow{2}{*}{$\begin{array}{l}\text { Previous } \\
\text { Quarter }\end{array}$} & \multirow{2}{*}{$\begin{array}{c}\text { Same } \\
\text { Quarter } \\
\text { Last Year }\end{array}$} & \multicolumn{2}{|c|}{$\begin{array}{c}\text { Current Quarter as \% change } \\
\text { from: }\end{array}$} \\
\hline & & & & & Previous Qtr & Same Qtr Last Year \\
\hline Tomatoes & 83 & $27 \%$ & 1 & 106 & - & $-22 \%$ \\
\hline Oranges & 39 & $13 \%$ & 1 & 38 & $2,966 \%$ & $3 \%$ \\
\hline Grapefruit & 36 & $12 \%$ & 1 & 34 & - & $4 \%$ \\
\hline Tomatoes, Plum Type & 25 & $8 \%$ & $<1$ & 29 & - & $-15 \%$ \\
\hline Peppers, Bell Type & 18 & $6 \%$ & - & 24 & - & $-23 \%$ \\
\hline Top 5 Total & 201 & $66 \%$ & 3 & 232 & $6,906 \%$ & $-13 \%$ \\
\hline Florida Total & 305 & $100 \%$ & 21 & 387 & $1,340 \%$ & $-21 \%$ \\
\hline
\end{tabular}

Source: AMS Transportation Services Division analysis of AMS Specialty Crops Program Market News data.

Any "-" in the table indicates no reported shipments during the quarter or a percentage change with an at- or near-zero denominator. Note: Table values may not conform exactly due to rounding. 
Figure 15: Florida Truck Rates (\$/Mile by Distance Travelled)

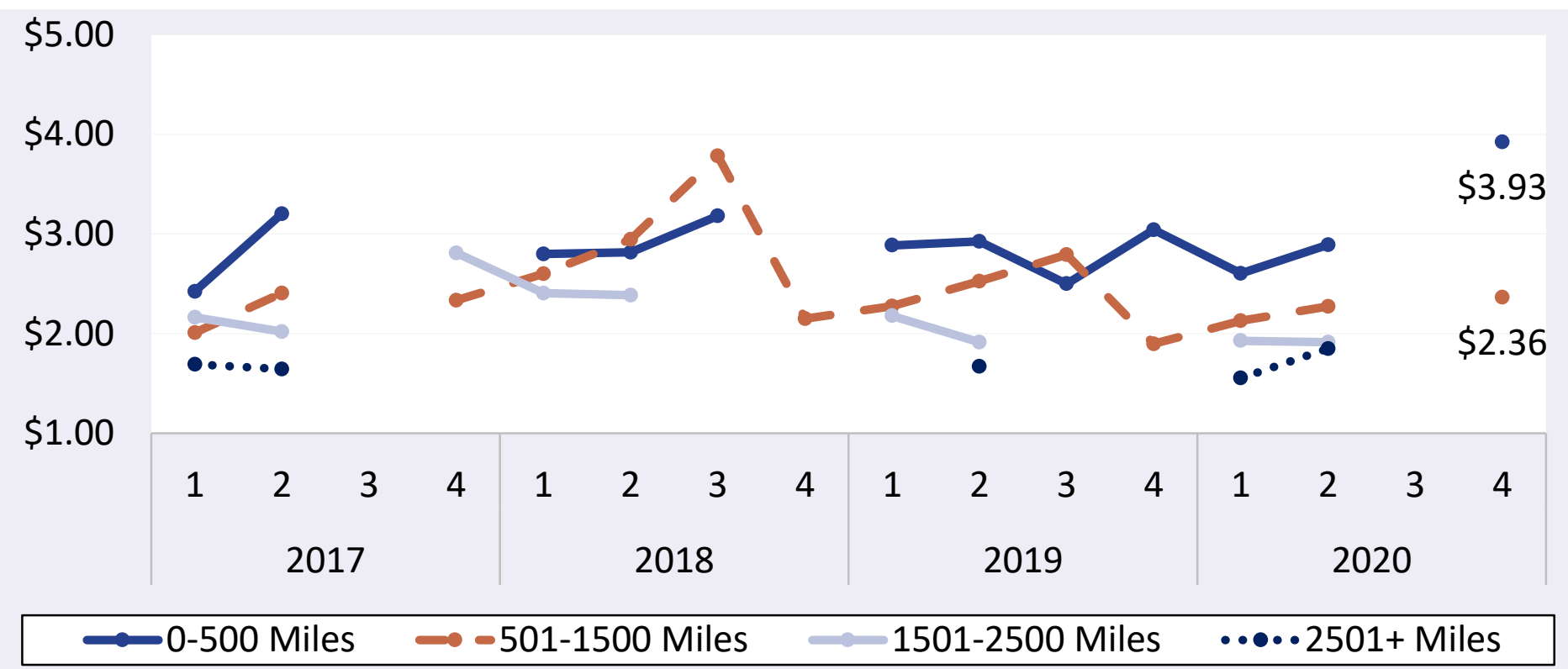

Source: AMS Transportation Services Division analysis of AMS Specialty Crops Program Market News data. Any gaps in the chart lines are the result of quarters with no reported data for the region.

Table 21: Florida Truck Overview (Availability Rating: 1=Surplus to 5=Shortage)

\begin{tabular}{|c|c|c|c|c|}
\hline Region/Reporting District & October & November & December & 4th Quarter \\
\hline Central \& South Florida & - & 3.75 & 4.2 & 3.98 \\
\hline West District Florida & 4 & 4.25 & - & 4.13 \\
\hline Regional Average Availability & 4 & 4 & 4.2 & 4.07 \\
\hline Diesel Fuel Price (\$)/gallon) & $\mathbf{2 . 3 2}$ & $\mathbf{2 . 3 4}$ & $\mathbf{2 . 4 9}$ & $\mathbf{2 . 3 8}$ \\
\hline
\end{tabular}

Source: AMS Transportation Services Division analysis of AMS Specialty Crops Program Market News data.

Any "-" in the table indicates no reported shipments during the quarter.

Note: Table values may not conform exactly due to rounding.

Note: AMS Specialty Crops Program defines regions by commodity, which may overlap in truck availability reporting. 


\section{Agricultural Refrigerated Truck Quarterly \\ Quarter 4, 2020}

Figure 16: Refrigerated Truck Availability Monthly Ratings for Florida

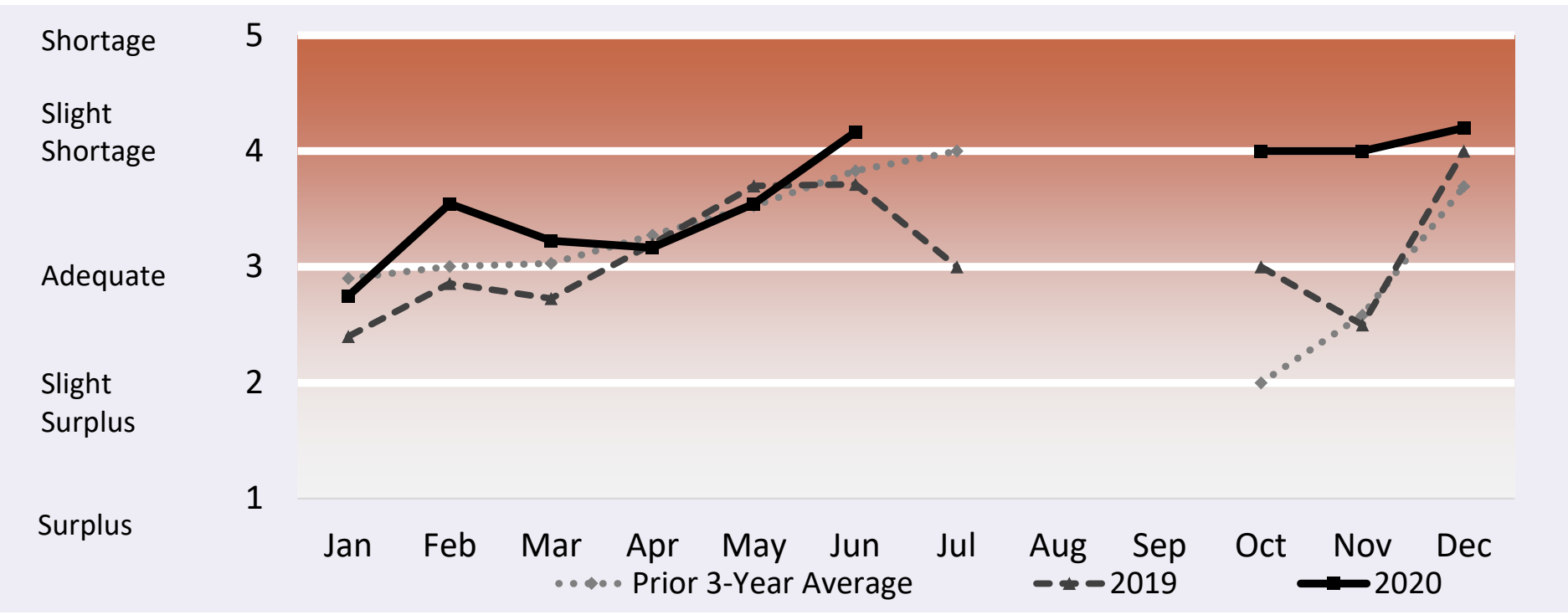

Source: AMS Transportation Services Division analysis of AMS Specialty Crops Program Market News data.

Any gaps in the chart lines are the result of quarters with no reported data for the region. 


\section{ADDITIONAL INFORMATION Terms and References}

\section{Data Sources}

This information is compiled from the weekly Specialty Crops Truck Rate Report by USDA, Agricultural Marketing Service (AMS), Specialty Crops Program, Market News Division. The website is: https://www.marketnews.usda.gov/mnp/fv-home.

\section{Regional Markets}

For the regional markets, some States are grouped into producing regions. The Pacific Northwest region includes Idaho, Oregon, and Washington. The Great Lakes region includes Michigan, Minnesota, and Wisconsin. The Southeast region includes North Carolina, South Carolina and Georgia.

\section{Shipment Volumes}

Truck shipments for all commodities and origins are not available. Those obtainable are reported, but should not be interpreted as representing complete movements of a commodity. Truck shipments from all States are collected at shipping points and include both interstate and intrastate movements. They are obtained from various sources, including Federal marketing orders, administrative committees, Federal State Inspection Service, and shippers. Volume amounts are represented in 10,000 pound units, or 1,000 10-lb packages but are converted to 1,000 tons for this report. Mexican border crossings through Arizona and Texas data is obtained from the Department of Homeland Security (DHS), U.S. Customs and Border and Protection (CBP) through USDA, AMS, Market News.

\section{Rates}

This information is compiled from the weekly Specialty Crops Truck Rate Report. Rates quoted represent open (spot) market rates that shippers or receivers pay depending on basis of sale, per load, including truck brokers fees for shipments in truck load volume to a single destination. Extra charges for delivery to terminal markets, multipickup and multidrop shipments are not included unless otherwise stated. Rates are based on the most usual loads in 48-53 foot trailers from the origin shipping area to the destination receiving city. In areas where rates are based on package rates, per load rates were derived by multiplying the package rate by the number of packages in the most usual load in a 48-53 foot trailer. Slightly cheaper rates will be reported during Quarters 2 and 3 as about 50 percent of onion shipments from California are hauled on open flatbed trailers. During Quarter 3, less than 20 percent of onions hauled from Washington, Idaho, and Oregon are on open flatbeds.

\section{Regional Rates}

Rate data for 10 destination markets are used to calculate average origin regional rates.

\section{National Rates}

The national rates reflect the average of the regional rates, separated by mileage category and weighted by volume between origin and destination. 


\section{Contact Us}

\section{Authors}

April Taylor, Coordinator; Analyst

April.Taylor@usda.gov, 202.720.7880

Jesse Gastelle, Analyst

Jesse.Gastelle@usda.gov, 202.690.1144

Kranti Mulik, Analyst

Kranti.Mulik@usda.gov, 202.756.2577

Matt Chang, Analyst

Matt.Chang@usda.gov, 202.720.0299

Patty Willkie, Specialty Crops Program and Market News Division Data

Patty.Willkie@usda.gov, 202.720.2175

To subscribe, please send an e-mail to April.Taylor@usda.gov. Printed copies are available upon request.

For assistance with accessibility issues related to this document, please e-mail SharonC.Williams@usda.gov.

\section{Related Websites}

USDA's Agricultural Transportation Open Data Platform

https://agtransport.usda.gov/

Specialty Crops Program

https://www.ams.usda.gov/about-ams/programs-offices/specialty-crops-program

Specialty Crops Truck Rate Report

https://mymarketnews.ams.usda.gov/filerepo/reports?field slug id value $=2375$

Specialty Crops Movement Reporting

https://www.marketnews.usda.gov/mnp/fv-report-config-step1?type=movement

Economic Research Service Vegetable and Pulses

https://www.ers.usda.gov/topics/crops/vegetables-pulses/

Economic Research Service Fruit and Tree Nuts

https://www.ers.usda.gov/topics/crops/fruit-tree-nuts.aspx

National Agricultural Statistics Service, Crops

https://www.nass.usda.gov/Statistics by Subject/index.php?sector=CROPS

Refrigerated Truck Quarterly Datasets

https://www.ams.usda.gov/services/transportation-analysis/agricultural-refrigerated-truck-quarterly-datasets

Protecting Perishable Foods During Transport by Truck and Rail

https://edis.ifas.ufl.edu/pdffiles/HS/HS132800.pdf

\section{Preferred Citation}

U.S. Department of Agriculture, Agricultural Marketing Service. Agricultural Refrigerated Truck Quarterly

Report. August 2021 Web. <https://dx.doi.org/10.9752/TS051.08-2021>

\section{Photo Credit}

Burt Barnes

USDA is an equal opportunity provider, employer, and lender. 